\title{
TWO-PARTICLE CLUSTER APPROXIMATION FOR ISING TYPE MODEL WITH ARBITRARY VALUE OF SPIN. CORRELATION FUNCTIONS OF BLUME-EMERY-GRIFFITHS MODEL
}

\author{
S.I.Sorokov, R.R.LEVITSKII, O.R.BARAN \\ Institute for Condensed Matter Physics \\ of the Ukrainian National Academy of Sciences \\ 1 Svientsitskii St., UA-290011 Lviv-11, Ukraine
}

Received January 25, 1997

\begin{abstract}
The Ising type model with arbitrary value of spin is investigated within the two-particle cluster approximation. For this model on hypercubic lattices the expressions for the pair correlation functions in $\vec{q}$-space are obtained. For Blume-Emery-Griffiths model $(S=1)$ on a simple cubic lattice with bilinear $K$, biquadratic $K^{\prime}$ interactions and single-ion anisotropy $D$, a projection of the phase diagram on $D / K-K^{\prime} / K$ plane is constructed. The temperature dependences of $\left\langle S^{z}\right\rangle,\left\langle\left(S^{z}\right)^{2}\right\rangle$ and pair correlation functions at $\vec{q}=0$ are calculated at various values of parameters $D / K$ and $K^{\prime} / K$.
\end{abstract}

\section{Introduction}

Among pseudospin systems studied theoretically, a great attention is paid to Ising-type models, that is, to systems with the Hamiltonian containing only $z$-component of pseudospin. That is so due to relative simplicity with which approximate calculations for these models can be carried out and tested and possibility of their application to a wide class of real objects. Thus, the Blume-Emery-Griffiths model (BEG) corresponding to the Isingtype model with $\eta=2$ (hereafter $S^{z}=-\eta,-\eta+2, \ldots, \eta-2, \eta$ ) was proposed for the investigation of phase transitions (PT) in $H e_{3}-H e_{4}$ mixture [1]. A general form of the Ising-type model with $\eta=2$ contains extra terms, as compared to the BEG model, like $S_{i}^{z}\left(S_{j}^{z}\right)^{2}$. It is used $[2,3]$ for description of external pressure influence on ferromagnetics $(\eta=1)$, for investigation of crystals with ferromagnetic $(\eta=1)$ impurities, three-component nonmagnetic alloys, two-component lattice liquids etc. The Ising-type model is proved to be useful in studies of tricritical behaviour of anisotropic ferromagnetics $\mathrm{FeCl}_{2}$ and $\mathrm{DAG}$, of pressure-induced structural phase transitions in $\mathrm{NH}_{4} \mathrm{Cl}$ and $\mathrm{KH}_{2} \mathrm{PO}_{4}[2,4]$.

For compounds described by pseudospin models with essential shortrange correlations, the cluster expansion method (CEM) [5-7] is the most natural many-particle generalization of the molecular field approximation (MFA). It should be mentioned that the CEM gives much better results at temperatures far from transition point. Within CEM, an infinite lattice is replaced with a cluster with a fixed number of pseudospins, the influence

(c) S.I.Sorokov, R.R.Levitskii, O.R.Baran, 1997

ISSN 0452-9910. Condensed Matter Physics 1997 No 9 (57-87) 
of rejected sites is taken into account as a single external field $\varphi(S)$, acting on boundary sites of a cluster. The first consistent formulation of the CEM which allows to determine corrections to free energy related to cluster interaction, was made in [8]. The first order of CEM is called cluster approximation.

Despite a number of papers where CEM is applied to calculation of physical characteristics of various substances, we are aware only of a few ones where pseudospin models with a spin value $\eta>1$ are considered. Thus, in [9] two-particle cluster approximation (TPCA) was applied to Heisenberg model with spin $\eta>1$, with the dependence of the variational field $\varphi$ on spin value being neglected. This neglecting gave qualitatively wrong results even for Ising-type model with $\eta=2[3]$. In [10] the BEG model was studied within TPCA. Even though the dependence $\varphi(S)$ was taken into account here, the coefficients of expansion of variational fields $\varphi$ in powers of pseudospin $\left(\varphi(S)=\varphi^{(1)}+\varphi^{(2)} S\right)$ were taken to be equal, up to constant terms, to $\langle S\rangle,\left\langle S^{2}\right\rangle$, what gave results close to those of MFA.

For investigation of thermodynamic properties of BEG model, other approximate methods also were widely used. In particular, in a series of papers [11-15], equation chains obtained on the basis of Callen identities are closed with the help of the simplest decoupling $\left\langle S_{1} S_{2} \ldots S_{k}\right\rangle \approx\left\langle S_{1}\right\rangle\left\langle S_{2}\right\rangle \ldots\left\langle S_{k}\right\rangle$. Calculated in such an approximation transition temperature $T_{c}$ for the case of $\eta=1$ [16] differs from results of numerical methods more significantly than the $T_{c}$ calculated in TPCA does.

The PT of the BEG model at negative values of single-ion anisotropy and positive values of bilinear and biquadratic interaction were studied in [17] within the constant-coupling approximation, which results correspond to the TPCA results. The three-dimensional phase diagram was constructed, and temperature dependences of dipole and quadrupolar moments were obtained for some values of Hamiltonian parameters. In [18] the Bethe approximation (results of this approximation also correspond to the TPCA results) was used for investigation of the BEG model at arbitrary values of single-ion anisotropy and biquadratic interaction. Particular attention was paid the case of antiferro biquadratic interaction. Obtained results were compared with those of Monte Carlo method. We also should mention the results of studies of BEG model within the high-temperature expansion [19] and Monte Carlo methods [20,21]. To our best knowledge, only thermodynamic properties of the considered models have been studied so far.

The goal of this work is to develope a two-particle cluster approximation for calculation of thermodynamic characteristics and correlations functions (CF) of Ising-type model with an arbitrary value of spin and investigate the Blume-Emery-Griffiths model within this approximation.

\section{Problem formulation}

We consider a pseudospin system with $S=S^{z}=(-\eta,-\eta+2, \ldots$, $\eta-2, \eta)$, described by the Hamiltonian

$$
\begin{gathered}
\mathcal{H}\left(\left\{h^{(\cdot)}\right\}\right)=-\beta H=\sum_{n=1}^{\eta} \sum_{i=1}^{N} h_{i}^{(n)} S_{i}^{n}+ \\
\frac{1}{2} \sum_{n=1}^{\eta} \sum_{m=1}^{\eta}\left[\sum_{i, \delta} K^{(n m)} S_{i}^{n} S_{i+\delta}^{m}+\sum_{i, j} J_{i j}^{(n m)} S_{i}^{n} S_{j}^{m}\right] .
\end{gathered}
$$


Here $K^{(n m)}$ and $J_{i j}^{(n m)}$ are the constants of short-range and long-range interactions, respectively; the notation $\mathcal{H}\left(\left\{h^{(\cdot)}\right\}\right)$ means that $\mathcal{H}$ is a function of $\left(h_{1}^{(1)}, \ldots, h_{N}^{(1)}, \ldots, h_{1}^{(\eta)}, \ldots, h_{N}^{(\eta)}\right)$. The factor $\beta=\left(k_{B} T\right)^{-1}$ occurring in $h_{i}^{(n)}, K^{(n m)}, J_{i j}^{(n m)}$ will be written explicitly only in some final formulas. As a particular case we consider Blume-Emery-Griffiths model $(\eta=2)$ with long-range interaction:

$$
\begin{gathered}
\mathcal{H}=\sum_{i=1}^{N}\left[\Gamma_{i} S_{i}+D_{i} S_{i}^{2}\right]+\frac{1}{2} \sum_{i, \delta}\left[K S_{i} S_{i+\delta}+K^{\prime} S_{i}^{2} S_{i+\delta}^{2}\right]+ \\
\frac{1}{2} \sum_{i, j}\left[J_{i j} S_{i} S_{j}+J_{i j}^{\prime} S_{i}^{2} S_{j}^{2}\right]
\end{gathered}
$$

Here $\Gamma_{i}$ is an external field, $D_{i}$ is a single-ion anisotropy, $K$ and $K^{\prime}$ are the constants of bilinear and biquadratic short-range interactions; $J_{i j}$ and $J_{i j}^{\prime}$ are those of long-range interactions.

Within the molecular field approximation in the long-range interaction, the Hamiltonian (2.1) can be expressed as

$$
\mathcal{H}\left(\left\{h^{(\cdot)}\right\}\right)={ }^{k} \mathcal{H}\left(\left\{\mathfrak{x}^{(\cdot)}\right\}\right)-\frac{1}{2} \sum_{n=1}^{\eta} \sum_{m=1}^{\eta} \sum_{i, j} J_{i j}^{(n m)}\left\langle S_{i}^{n}\right\rangle\left\langle S_{j}^{m}\right\rangle .
$$

The following notation for the reference Hamiltonian is used

$$
\begin{aligned}
{ }^{k} \mathcal{H}\left(\left\{æ^{(\cdot)}\right\}\right) & =\sum_{n=1}^{\eta} \sum_{i=1}^{N} æ_{i}^{(n)} S_{i}^{n}+\frac{1}{2} \sum_{n=1}^{\eta} \sum_{m=1}^{\eta} \sum_{i, \delta} K^{(n m)} S_{i}^{n} S_{i+\delta}^{m}, \\
æ_{i}^{(n)} & =h_{i}^{(n)}+\sum_{m=1}^{\eta} \sum_{j=1}^{N} J_{i j}^{(n m)}\left\langle S_{j}^{m}\right\rangle
\end{aligned}
$$

The function $\mathcal{F}\left(\left\{h^{(\cdot)}\right\}\right)$ (logarithm of the partition function) within the MFA in the long-range interaction reads:

$\mathcal{F}\left(\left\{h^{(\cdot)}\right\}\right)=\ln \operatorname{Sp}_{\{S\}} \mathrm{e}^{\mathcal{H}}={ }^{k} \mathcal{F}\left(\left\{æ^{(\cdot)}\right\}\right)-\frac{1}{2} \sum_{n=1}^{\eta} \sum_{m=1}^{\eta} \sum_{i, j} J_{i j}^{(n m)}\left\langle S_{i}^{n}\right\rangle\left\langle S_{j}^{m}\right\rangle$,

where ${ }^{k} \mathcal{F}\left(\left\{\mathfrak{x}^{(\cdot)}\right\}\right)$ is a logarithm of the partition function of the reference system (2.4).

Correlation functions (cumulant averages of spin operators calculated with the Gibbs' distribution with $\mathcal{H}$ ) of the considered model will be evaluated as:

$$
\left\langle\left(S_{i_{1}}^{n_{1}}\right)^{\nu_{1}} \ldots\left(S_{i_{k}}^{n_{k}}\right)^{\nu_{k}}\right\rangle^{c}=\frac{\delta^{\nu_{1}}}{\delta\left(h_{i_{1}}^{\left(n_{1}\right)}\right)^{\nu_{1}}} \cdots \frac{\delta^{\nu_{k}}}{\delta\left(h_{i_{k}}^{\left(n_{k}\right)}\right)^{\nu_{k}}} \mathcal{F}\left(\left\{h^{(\cdot)}\right\}\right)
$$

and CFs of the reference system as:

$$
{ }^{k}\left\langle\left(S_{i_{1}}^{n_{1}}\right)^{\nu_{1}} \ldots\left(S_{i_{k}}^{n_{k}}\right)^{\nu_{k}}\right\rangle^{c}=\frac{\delta^{\nu_{1}}}{\delta\left(æ_{i_{1}}^{\left(n_{1}\right)}\right)^{\nu_{1}}} \cdots \frac{\delta^{\nu_{k}}}{\delta\left(æ_{i_{k}}^{\left(n_{k}\right)}\right)^{\nu_{k}}}{ }^{k} \mathcal{F}\left(\left\{æ^{(\cdot)}\right\}\right) .
$$


From the expression for the $\mathcal{F}$-function $(2.6)$ we can easily derive some relations between single-site CFs of the reference system (2.4) and those of the general system within the MFA in long-range interactions (2.3):

$$
\left\langle S_{i}^{n}\right\rangle=\frac{\delta}{\delta\left(h_{i}^{(n)}\right)} \mathcal{F}\left(\left\{h^{(\cdot)}\right\}\right)=\frac{\delta}{\delta\left(æ_{i}^{(n)}\right)}{ }^{k} \mathcal{F}\left(\left\{æ^{(\cdot)}\right\}\right)={ }^{k}\left\langle S_{i}^{n}\right\rangle .
$$

For the sake of simplicity, we present relations between pair correlation functions for the BEG model (2.2) only. These relations can be obtained from $(2.9) \quad\left(n=1,2 ; \quad J=J^{(11)}, \quad J^{\prime}=J^{(22)}, \quad J^{(12)}=J^{(21)}=0\right)$. Using matrix notations in the indices $i, j$ and performing Fourier transformation, we get a system of four equations relating $\left\langle S^{n} S^{m}\right\rangle_{\vec{q}}^{c}$ and ${ }^{k}\left\langle S^{n^{\prime}} S^{m^{\prime}}\right\rangle_{\vec{q}}^{c}$. In a matrix form it reads

$$
\begin{gathered}
\hat{b}(\vec{q})={ }^{k} \widehat{b}(\vec{q})+{ }^{k} \widehat{b}(\vec{q})\left(\begin{array}{cc}
J_{\vec{q}} & 0 \\
0 & J_{\vec{q}}^{\prime}
\end{array}\right) \hat{b}(\vec{q}), \\
\widehat{b}(\vec{q})=\left(\begin{array}{cc}
\langle S S\rangle_{\vec{q}}^{c} & \left\langle S S^{2}\right\rangle_{\vec{q}}^{c} \\
\left\langle S^{2} S\right\rangle_{\vec{q}}^{c} & \left\langle S^{2} S^{2}\right\rangle_{\vec{q}}^{c}
\end{array}\right) ;{ }^{k} \widehat{b}(\vec{q})=\left(\begin{array}{cc}
{ }^{k}\langle S S\rangle_{\vec{q}}^{c} & { }^{k}\left\langle S S^{2}\right\rangle_{\vec{q}}^{c} \\
{ }^{k}\left\langle S^{2} S\right\rangle_{\vec{q}}^{c} & { }^{k}\left\langle S^{2} S^{2}\right\rangle_{\vec{q}}^{c}
\end{array}\right) .
\end{gathered}
$$

From equation (2.10) one can easily find the pair CFs of the BEG model expressed in terms of pair CFs of the reference system, the long-range interactions taken into account in the MFA.

\section{Two particle cluster approximation in short-range inter- actions}

In this section we consider the reference pseudospin system with Hamiltonian (2.4). Let us divide the lattice into two-particle clusters. As $\sum_{n=1}^{\eta}{ }^{r} \varphi_{i}^{(n)} S_{i}^{n}$ we denote an operator of the effective field created by the site $r$ and acting on the site $i$, provided that the site $r$ is a nearest neighbour of the site $i\left(r \in \pi_{i}\right)$. Apparently, when the lattice is divided into the twoparticle clusters, the number of fields acting on the given site is equal to the number of the nearest neighbours $z$. We transfer from summing over lattice sites to summing over clusters [22]:

$$
\begin{gathered}
\frac{1}{2} \sum_{i, \delta} K^{(n m)} S_{i}^{n} S_{i+\delta}^{m}=\sum_{(1,2)} K^{(n m)} S_{1}^{n} S_{2}^{m} \\
\sum_{i} \sum_{r \in \pi_{i}}{ }^{r} \varphi_{i}^{(n)} S_{i}^{n}=\sum_{(1,2)}\left({ }^{2} \varphi_{1}^{(n)} S_{1}^{n}+{ }^{1} \varphi_{2}^{(n)} S_{2}^{n}\right) .
\end{gathered}
$$

Using (3.1), we can write the reference Hamiltonian (2.4) in the form

$$
{ }^{k} \mathcal{H}\left(\left\{æ^{(\cdot)}\right\},\left\{\varphi^{(\cdot)}\right\}\right)=\sum_{1} \mathcal{H}_{1}\left(\left\{\tilde{æ}_{1}^{(\cdot)}\right\}\right)+\sum_{(1,2)} U_{12},
$$

provided that

$$
\begin{aligned}
& \mathcal{H}_{1}\left(\left\{\tilde{\mathfrak{X}}_{1}^{(\cdot)}\right\}\right)=\sum_{n=1}^{\eta} \tilde{\mathfrak{x}}_{1}^{(n)} S_{1}^{n} ; \quad \tilde{æ}_{1}^{(n)}=\mathfrak{x}_{1}^{(n)}+\sum_{r \in \pi_{1}}{ }^{r} \varphi_{1}^{(n)}, \\
& U_{12}=\sum_{n=1}^{\eta}\left(-{ }^{2} \varphi_{1}^{(n)} S_{1}^{n}-{ }^{1} \varphi_{2}^{(n)} S_{2}^{n}+\sum_{m=1}^{\eta} K^{(n m)} S_{1}^{n} S_{2}^{m}\right) .
\end{aligned}
$$


Notation $\mathcal{H}_{1}\left(\left\{\tilde{\mathfrak{x}}_{1}^{(\cdot)}\right\}\right)$ means that $\mathcal{H}_{1}$ is a function of $\left(\tilde{\mathfrak{x}}_{1}^{(1)}, \ldots, \tilde{\mathfrak{x}}_{1}^{(\eta)}\right)$.

$$
\begin{aligned}
& \text { For }{ }^{k} \mathcal{F}\left(\left\{\mathfrak{x}^{(\cdot)}\right\},\left\{\varphi^{(\cdot)}\right\}\right) \text { we have: } \\
& { }^{k} \mathcal{F}\left(\left\{\mathfrak{x}^{(\cdot)}\right\},\left\{\varphi^{(\cdot)}\right\}\right)=\ln \operatorname{Sp}_{\{S\}} \exp \left[{ }^{k} \mathcal{H}\left(\left\{æ^{(\cdot)}\right\},\left\{\varphi^{(\cdot)}\right\}\right)\right]= \\
& \sum_{1} F_{1}\left(\left\{\tilde{x}_{1}^{(\cdot)}\right\}\right)+\ln \left\langle\exp \left(\sum_{(1,2)} U_{12}\right)\right\rangle_{\rho_{0}} ; \\
& \langle A\rangle_{\rho_{0}}=\operatorname{Sp}_{\{S\}}\left(\rho_{0}(\{S\}) \cdot A\right) ; \quad \rho_{0}(\{S\})=\prod_{1} \rho_{1}\left(S_{1}\right)=\prod_{1} \frac{\exp \left(\mathcal{H}_{1}\right)}{Z_{1}} .
\end{aligned}
$$

Here we introduced the following notations for the single-particle $F_{1}$-function:

$$
F_{1}\left(\left\{\tilde{\mathfrak{x}}_{1}^{(\cdot)}\right\}\right)=\ln Z_{1}\left(\left\{\tilde{\mathfrak{x}}_{1}^{(\cdot)}\right\}\right) ; \quad Z_{1}\left(\left\{\tilde{\mathfrak{x}}_{1}^{(\cdot)}\right\}\right)=\operatorname{Sp}_{S_{1}} \exp \left[\mathcal{H}_{1}\left(\left\{\tilde{\mathfrak{x}}_{1}^{(\cdot)}\right\}\right)\right] .
$$

Let us restrict ourselves to the first order of the cluster expansion [22]. Then ${ }^{k} \mathcal{F}$-function can be written as a sum of single-particle and two-particle intracluster $F$-functions

$$
\begin{array}{r}
{ }^{k} \mathcal{F}\left(\left\{\mathfrak{x}^{(\cdot)}\right\},\left\{\varphi^{(\cdot)}\right\}\right)=\sum_{1} F_{1}\left(\left\{\tilde{\mathfrak{x}}_{1}^{(\cdot)}\right\}\right)+\sum_{(1,2)} \ln \left\langle\exp \left(U_{12}\right)\right\rangle_{\rho_{0}}= \\
(1-z) \sum_{1} F_{1}\left(\left\{\tilde{\mathfrak{x}}_{1}^{(\cdot)}\right\}\right)+\frac{1}{2} \sum_{1,2} F_{12}\left(\left\{{ }^{2} \tilde{\mathfrak{x}}_{1}^{(\cdot)}\right\},\left\{{ }^{1} \tilde{\mathfrak{x}}_{2}^{(\cdot)}\right\}\right),
\end{array}
$$

where the two-particle $F_{12}$-function reads:

$$
\begin{aligned}
& F_{12}\left(\left\{{ }^{2} \tilde{\dddot{x}}_{1}^{(\cdot)}\right\},\left\{{ }^{1} \tilde{\mathfrak{x}}_{2}^{(\cdot)}\right\}\right)=\ln Z_{12}\left(\left\{{ }^{2} \tilde{\mathfrak{x}}_{1}^{(\cdot)}\right\},\left\{{ }^{1} \tilde{\mathfrak{x}}_{2}^{(\cdot)}\right\}\right)= \\
& \quad \ln \operatorname{Sp}_{S_{1}, S_{2}} \exp \left(\mathcal{H}_{12}\right), \\
& \mathcal{H}_{12}\left(\left\{{ }^{2} \tilde{\mathfrak{x}}_{1}^{(\cdot)}\right\},\left\{{ }^{1} \tilde{\mathfrak{x}}_{2}^{(\cdot)}\right\}\right)=\mathcal{H}_{1}\left(\left\{\tilde{\mathfrak{x}}_{1}^{(\cdot)}\right\}\right)+\mathcal{H}_{2}\left(\left\{\tilde{\mathfrak{x}}_{2}^{(\cdot)}\right\}\right)+U_{12}= \\
& \quad \sum_{n=1}^{\eta}\left({ }^{2} \tilde{\mathfrak{x}}_{1}^{(n)} S_{1}^{n}+{ }^{1} \tilde{\mathfrak{x}}_{2}^{(n)} S_{2}^{n}+\sum_{m=1}^{\eta} K^{(n m)} S_{1}^{n} S_{2}^{m}\right), \\
& { }^{1} \tilde{\mathfrak{x}}_{2}^{(n)}=\tilde{\mathfrak{x}}_{2}^{(n)}-{ }^{1} \varphi_{2}^{(n)}=\mathfrak{x}_{2}^{(n)}+\sum_{\substack{r \in \pi_{2} \\
r \neq 1}}^{r} \varphi_{2}^{(n)} .
\end{aligned}
$$

Let us consider now the ${ }^{k} \mathcal{F}$-function. Using the method, proposed in [23] for the case $\eta=1$ we can obtain equations for $\left\langle S_{1}^{n}\right\rangle={ }^{k}\left\langle S_{1}^{n}\right\rangle$ and cluster fields ${ }^{r} \varphi_{1}^{(n)}$. From (2.8) and (2.9) we get:

$$
\left\langle S_{1}^{n}\right\rangle=\frac{\partial^{k} \mathcal{F}}{\partial x_{1}^{(n)}}+\sum_{i} \sum_{r \in \pi_{i}} \sum_{m} \frac{\partial^{k} \mathcal{F}}{\partial{ }^{r} \varphi_{i}^{(m)}} \cdot \frac{\delta^{r} \varphi_{i}^{(m)}}{\delta x_{1}^{(n)}} .
$$

Taking into account the fact that ${ }^{k} \mathcal{F}$-function (3.8) is a sum of single-particle and two-particle $F$-functions and making use of the notations (3.3) and 
(3.11), we get the following expressions for the partial derivatives of ${ }^{k} \mathcal{F}$ functions:

$$
\begin{gathered}
\left.\frac{\partial^{k} \mathcal{F}}{\partial æ_{1}^{(n)}}=(1-z) \cdot F_{1}\left(\begin{array}{c}
1 \\
n
\end{array}\right)+\sum_{r \in \pi_{1}} F_{1 r}\left(\begin{array}{l}
1 \\
n
\end{array}\right)\right) \\
\frac{\partial^{k} \mathcal{F}}{\partial{ }^{r_{1}} \varphi_{i}^{(n)}}=(1-z) \cdot F_{i}\left(\begin{array}{c}
1 \\
n
\end{array}\right)+\sum_{r \in \pi_{i}} F_{i r}\left(\begin{array}{c}
1 \\
n
\end{array}\right)-F_{i r_{1}}\left(\begin{array}{c}
1 \\
n
\end{array}\right) ; \quad\left(r_{1} \in \pi_{i}\right) .
\end{gathered}
$$

Here we use the notations for the partial derivatives of the single-particle and two-particle $F$-functions - the single-site and pair intracluster CFs:

$$
\begin{aligned}
& F_{i}\left(\begin{array}{cccc}
\nu_{1} \\
n_{1}
\end{array}\left|\begin{array}{ccc}
\nu_{2} \\
n_{2}
\end{array}\right| \cdots \quad \begin{array}{l}
\nu_{k} \\
n_{k}
\end{array} \mid\left\{\tilde{\mathfrak{x}}_{i}^{(\cdot)}\right\}\right)=\frac{\partial^{\nu_{1}}}{\partial\left(\tilde{\mathfrak{x}}_{i}^{\left(n_{1}\right)}\right)^{\nu_{1}}} \cdots \frac{\partial^{\nu_{k}}}{\partial\left(\tilde{\mathfrak{x}}_{i}^{\left(n_{k}\right)}\right)^{\nu_{k}}} F_{i}\left(\left\{\tilde{\mathfrak{x}}_{i}^{(\cdot)}\right\}\right),
\end{aligned}
$$

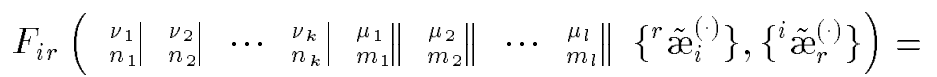

$$
\begin{aligned}
& \left.\frac{\partial^{\nu_{1}}}{\partial\left({ }^{r} \tilde{\mathfrak{x}}_{i}^{\left(n_{1}\right)}\right)^{\nu_{1}}} \cdots \frac{\partial^{\nu_{k}}}{\partial\left({ }^{r} \tilde{\mathfrak{x}}_{i}^{\left(n_{k}\right)}\right)^{\nu_{k}}} \frac{\partial^{\mu_{1}}}{\partial\left({ }^{i} \tilde{\mathfrak{x}}_{r}^{\left(m_{1}\right)}\right)^{\mu_{1}}} \cdots \frac{\partial^{\mu_{l}}}{\partial\left({ }^{i} \tilde{\mathfrak{æ}}_{r}^{\left(m_{l}\right)}\right)^{\mu_{l}}} F_{i r}\left({ }^{r} \tilde{\mathfrak{x}}_{i}^{(\cdot)}\right\},\left\{{ }^{i} \tilde{\mathfrak{x}}_{r}^{(\cdot)}\right\}\right),
\end{aligned}
$$

On the basis of (2.9), (3.3), (3.11), (3.12), (3.13) and (3.14) taking into account the condition of an extremum of ${ }^{k} \mathcal{F}$-function with respect to ${ }^{r} \varphi_{i}^{(n)}$

$$
\frac{\partial^{k} \mathcal{F}}{\partial^{r} \varphi_{i}^{(n)}}=0
$$

we can easily get the system of $(z+1) \eta N$ equations for ${ }^{r} \varphi_{i}^{(n)}$ and $\left\langle S_{i}^{n}\right\rangle \quad(n=$ $1, \ldots, \eta ; i=1, \ldots, N)$.

$$
\begin{aligned}
& \left\langle S_{1}^{n}\right\rangle=F_{1}\left(\begin{array}{c}
1 \\
n
\end{array} \mid\left\{\tilde{\mathfrak{x}}_{1}^{(\cdot)}\right\}\right) \\
& F_{1}\left(\begin{array}{l}
1 \\
n
\end{array} \mid\left\{\tilde{\mathfrak{x}}_{1}^{(\cdot)}\right\}\right)=F_{1 r}\left(\begin{array}{c}
1 \\
n
\end{array} \mid\left\{{ }^{r} \tilde{\mathfrak{x}}_{1}^{(\cdot)}\right\},\left\{{ }^{1} \tilde{\mathfrak{x}}_{r}^{(\cdot)}\right\}\right)
\end{aligned}
$$

On the other hand, from (3.7), (3.9), and definitions of intracluster averages

$$
\langle A\rangle_{\rho_{1}}=\operatorname{Sp}_{S_{1}}\left[\rho_{1}\left(S_{1}\right) \cdot A\right] ; \quad\langle A\rangle_{\rho_{1 r}}=\operatorname{Sp}_{S_{1}, S_{r}}\left[\rho_{1 r}\left(S_{1}, S_{r}\right) \cdot A\right],
$$

we obtain the relations for the single-site intracluster CFs

$$
\left.F_{1}\left(\begin{array}{l}
1 \\
n
\end{array}\right)=\left\langle S_{1}^{n}\right\rangle_{\rho_{1}} ; \quad F_{1 r}\left(\begin{array}{c}
1 \\
n
\end{array}\right)\right)=\left\langle S_{1}^{n}\right\rangle_{\rho_{1 r}},
$$

the quantities are averaged with the intracluster single-particle and twoparticle density matrices

$$
\begin{aligned}
& \rho_{1}\left(S_{1}\right)=\frac{\exp \left[\mathcal{H}_{1}\left(\left\{\tilde{\mathfrak{x}}_{1}^{(\cdot)}\right\}\right)\right]}{Z_{1}\left(\left\{\tilde{\mathfrak{x}}_{1}^{(\cdot)}\right\}\right)} ; \\
& \rho_{1 r}\left(S_{1}, S_{r}\right)=\frac{\exp \left[\mathcal{H}_{1 r}\left(\left\{^{r} \tilde{\mathfrak{x}}_{1}^{(\cdot)}\right\},\left\{{ }^{1} \tilde{\mathfrak{x}}_{r}^{(\cdot)}\right\}\right)\right]}{Z_{1 r}\left(\left\{{ }^{r} \tilde{\mathfrak{x}}_{1}^{(\cdot)}\right\},\left\{{ }^{1} \tilde{\mathfrak{x}}_{r}^{(\cdot)}\right\}\right)} .
\end{aligned}
$$


Hence, from the condition of ${ }^{k} \mathcal{F}$-function extremum we have derived (see (3.18), (3.19)) the system of $z \eta N$ equations

$$
\left\langle S_{1}^{n}\right\rangle_{\rho_{1}}=\left\langle S_{1}^{n}\right\rangle_{\rho_{1 r}} ; \quad(n=1, \ldots, \eta) .
$$

As can be seen from definition (3.20), the system of $z \eta N$ equations (3.23) is equivalent to $z \eta N$ independent relations between the density matrices

$$
\rho_{1}\left(S_{1}\right)=\operatorname{Sp}_{S_{r}}\left[\rho_{1 r}\left(S_{1}, S_{r}\right)\right] ; \quad S=(-\eta,-\eta+2, \ldots, \eta-2, \eta) .
$$

Among $z(\eta+1) N$ relations (3.24), only $z \eta N$ are independent, for $z N$ conditions $\rho_{1}\left(S_{1}\right), \rho_{1 r}\left(S_{1}, S_{r}\right)$ are obeyed identically as follows from the form of $\operatorname{Sp}_{S_{1}}\left[\rho_{1}\left(S_{1}\right)\right]=\operatorname{Sp}_{S_{1}, S_{r}}\left[\rho_{1 r}\left(S_{1}, S_{r}\right)\right]=1$. Hence, in the TPCA, the condition of ${ }^{k} \mathcal{F}$-function extremum yields the relations (3.24) between the intracluster density matrices.

Let us note that in uniform fields $h_{i}^{(n)}\left(h_{i}^{(n)}=h^{(n)},\left\langle S_{i}^{n}\right\rangle=\left\langle S^{n}\right\rangle\right.$, $\left.x_{i}^{(n)}=æ^{(n)}\right)$, that is, when

$$
\begin{aligned}
& { }^{r} \tilde{\mathfrak{X}}_{1}^{(n)}=\tilde{\tilde{æ}}^{(n)}=\mathfrak{æ}^{(n)}+(z-1) \varphi^{(n)} ; \quad \tilde{æ}_{1}^{(n)}=\tilde{æ}^{(n)}=\varlimsup^{(n)}+z \varphi^{(n)} ; \\
& \mathfrak{x}^{(n)}=h^{(n)}+\sum_{m=1}^{\eta} J_{0}^{(n m)}\left\langle S^{m}\right\rangle ; \quad J_{0}^{(n m)}=J^{(n m)}(\vec{q}=0),
\end{aligned}
$$

relations (3.18) and (3.19) form the system of $2 \eta$ equations for $\varphi^{(n)}$ and $\left\langle S^{n}\right\rangle$. When the long-range interactions are absent $\left(J_{0}^{(n m)}=0\right)$ and the fields are uniform, we have the system of $\eta$ equations (3.19) for the cluster fields $\varphi^{(n)}$ and $\eta$ expressions (3.18) for the single-site CFs $\left\langle S^{n}\right\rangle$.

Let us briefly consider the proposed by us [3] method of calculation of pair CFs of the reference system with an arbitrary value of $\eta$, which is based on the method developed in [23] for the case $\eta=1$. From (2.8) and (3.18) one can obtain an expression for pair CFs of the reference system

$$
{ }^{k} b_{12}^{(n m)}={ }^{k}\left\langle S_{1}^{n} S_{2}^{m}\right\rangle^{c}=\sum_{k=1}^{\eta} F_{1}\left(\begin{array}{ll}
1 \\
n
\end{array}\left|\begin{array}{l}
1 \\
k
\end{array}\right|\right) \cdot \frac{\delta \tilde{\mathfrak{x}}_{1}^{(k)}}{\delta \mathfrak{x}_{2}^{(m)}} .
$$

Since evaluation of $F_{1}\left(\begin{array}{ll}1 \\ n\end{array}\left|\begin{array}{l}1 \\ k\end{array}\right|\right)$ for a particular system is straightforward, we only have to find an equation for $\delta \tilde{x}_{1}^{(k)} / \delta \mathfrak{x}_{2}^{(m)}$.

Let us introduce the notations

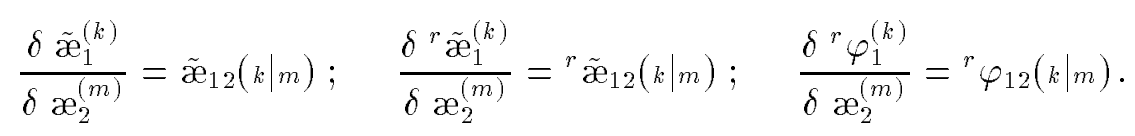

Differentiating both sides of equation (3.19) with respect to $x_{2}^{(m)}$, and taking into account the relation

$$
{ }^{r} \tilde{\dddot{x}}_{12}\left(\left.k\right|_{m}\right)=\tilde{\dddot{1}}_{12}\left(\left.k\right|_{m}\right)-{ }^{r} \varphi_{12}\left(\left.k\right|_{m}\right)
$$

(see (3.11)), we get

$$
\begin{aligned}
\sum_{k=1}^{\eta} F_{1}\left(\begin{array}{ll}
1 \\
n
\end{array} \mid \begin{array}{l}
k \\
k
\end{array}\right) \cdot \tilde{\mathfrak{X}}_{12}(k \mid m)= & \sum_{k=1}^{\eta} F_{1 r}\left(\begin{array}{ll}
1 \\
n
\end{array}\left|\begin{array}{l}
k \\
k
\end{array}\right|\right)\left[\tilde{\mathfrak{X}}_{12}(k \mid m)-{ }^{r} \varphi_{12}(k \mid m)\right]+ \\
& \sum_{k=1}^{\eta} F_{1 r}\left(\begin{array}{ll}
1 \\
n
\end{array}\left|\begin{array}{l}
1 \\
k
\end{array}\right|\right)\left[\tilde{\mathfrak{x}}_{r 2}(k \mid m)-{ }^{1} \varphi_{r 2}(k \mid m)\right],
\end{aligned}
$$


which can be transformed to

$$
\left[\widehat{F}_{1 r}^{(20)}+\widehat{F}_{1}^{(2)}\right] \cdot \widehat{\tilde{\dddot{ஜ}}}_{12}+\widehat{F}_{1 r}^{(11)} \cdot \widehat{\tilde{\dddot{ஜ}}}_{r 2}=\widehat{F}_{1 r}^{(20)} \cdot{ }^{r} \widehat{\varphi}_{12}+\widehat{F}_{1 r}^{(11)} \cdot{ }^{1} \widehat{\varphi}_{r 2} \cdot
$$

Here we use the notations:

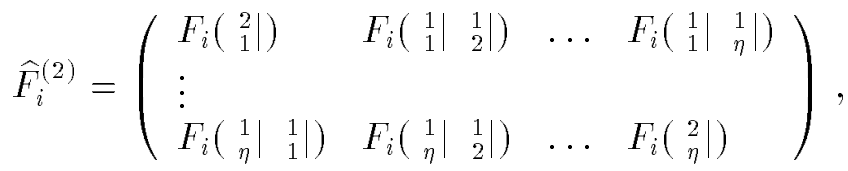

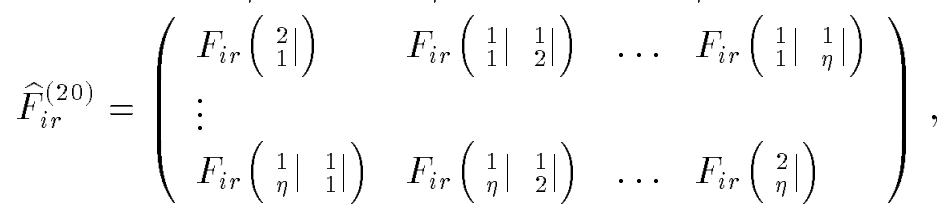

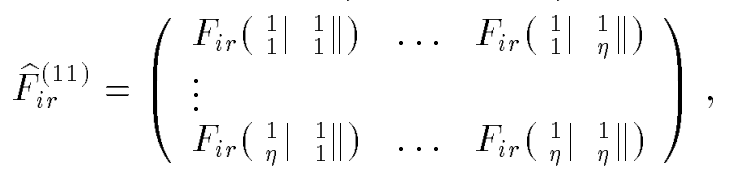

$$
\begin{aligned}
& \widehat{\tilde{\dddot{a}}}_{i r}=\left(\begin{array}{lll}
\tilde{\dddot{x}}_{i r}(1 \mid 1) & \ldots & \tilde{\mathfrak{x}}_{i r}\left(\left.1\right|_{\eta}\right) \\
\vdots & & \\
\tilde{\mathfrak{x}}_{i r}(\eta \mid 1) & \ldots & \tilde{\mathfrak{x}}_{i r}(\eta \mid \eta)
\end{array}\right) \text {. }
\end{aligned}
$$

Let us note that equations (3.30) contain unknown variables ${ }^{r} \widehat{\varphi}_{12}$ and ${ }^{1} \widehat{\varphi}_{r 2}$, for which we need to derive an independent equation. Using the relations

$$
F_{1}\left(\begin{array}{l}
2 \\
n
\end{array}\right)=F_{1 r}\left(\begin{array}{l}
2 \\
n
\end{array}\right) ; \quad F_{1}\left(\begin{array}{ll}
1 \\
n
\end{array} \mid \begin{array}{l}
1 \\
m
\end{array}\right)=F_{1 r}\left(\begin{array}{l}
1 \\
n
\end{array} \mid \begin{array}{c}
1 \\
m
\end{array}\right),
$$

following from the relations between the density matrices (3.24) and introducing the notations

$$
\widehat{f}_{1 r}=\left[\widehat{F}_{1 r}^{(20)}\right]^{-1} \cdot \widehat{F}_{1 r}^{(11)},
$$

we rewrite (3.30) in the form

$$
{ }^{r} \widehat{\varphi}_{12}+\widehat{f}_{1 r} \cdot{ }^{1} \widehat{\varphi}_{r 2}=\widehat{f}_{1 r} \cdot \widehat{\tilde{\dddot{x}}}_{r 2} .
$$

Exchanging indices $r \rightleftharpoons 1$, we obtain

$$
\widehat{f}_{r 1} \cdot{ }^{r} \widehat{\varphi}_{12}+{ }^{1} \widehat{\varphi}_{r 2}=\widehat{f}_{r 1} \cdot \widehat{\tilde{\dddot{\varkappa}}}_{12} \cdot
$$

(3.34) and (3.35) are the system of equations for ${ }^{r} \widehat{\varphi}_{12}$ and ${ }^{1} \widehat{\varphi}_{r 2}$. Carrying out summation over $r \in \pi_{1}$ in (3.34) and considering the fact that

$$
\sum_{r \in \pi_{1}}{ }^{r} \widehat{\varphi}_{12}=\widehat{\tilde{\dddot{x}}}_{12}-\widehat{\mathfrak{x}}_{12}=\widehat{\tilde{\dddot{\aleph}}}_{12}-\delta_{12} \cdot \hat{1},
$$

we can obtain from $(3.34),(3.35)$ a closed equation for $\widehat{\tilde{\dddot{x}}}_{12}$.

$$
\widehat{Q}_{11} \cdot \widehat{\tilde{\tilde{Q}}}_{12}=\delta_{12} \cdot \hat{1}+\sum_{r \in \pi_{1}} \widehat{f}_{1 r} \cdot \widehat{d}_{r 1 r}^{-1} \cdot \widehat{\tilde{\tilde{x}}}_{r 2} \cdot \pi_{1 r}
$$


Here we use the notations:

$$
\widehat{Q}_{11}=\widehat{1}+\sum_{r \in \pi_{1}} \widehat{f}_{1 r} \cdot \widehat{d}_{r 1 r}^{-1} \cdot \widehat{f}_{r 1} ; \quad \widehat{d}_{r 1 r}=\widehat{1}-\widehat{f}_{r 1} \cdot \widehat{f}_{1 r} ; \quad \pi_{1 r}=\left\{\begin{array}{ll}
1, & r \in \pi_{1} \\
0, & r \notin \pi_{1}
\end{array} .\right.
$$

In uniform fields the following relations hold:

$$
\widehat{\tilde{\dddot{x}}}_{12}=\widehat{\tilde{\tilde{x}}}(1-2) ; \quad \widehat{f}_{1 r}=\widehat{f}(1-r)=\widehat{f} ; \quad \widehat{d}_{r 1 r}=\widehat{d}(1-r)=\widehat{d}=\widehat{1}-\widehat{f}^{2} .
$$

Performing in (3.37) a Fourier transformation, the fields being uniform, and solving the obtained equation, we find $\widehat{\tilde{x}}(\vec{q})$

$$
\widehat{\widetilde{æ}}(\vec{q})=\left[z \cdot \hat{1}-(z-1)(\hat{1}+\hat{f})+z(\hat{1}-\hat{f})^{-1} \cdot \hat{f} \cdot \Theta(\vec{q})\right]^{-1} \cdot(\hat{1}+\hat{f}),
$$

where for a hypercubic lattice

$$
\Theta(\vec{q})=1-\frac{\pi(\vec{q})}{z}=\frac{2}{d} \sum_{i=1}^{d} \sin ^{2}\left(\frac{q_{i} \cdot a}{2}\right) .
$$

$d=z / 2$ is the lattice dimension, $\pi(\vec{q})$ is the Fourier transform of $\pi_{1 r}$.

Using the matrix form of (3.26) and performing a Fourier transformation, taking into account (3.40), we get the following relation for pair CFs of the reference system

$$
\begin{aligned}
{ }^{k} \widehat{b}(\vec{q})=\widehat{F}_{1}^{(2)} \cdot \widehat{\tilde{x}}(\vec{q})= & \frac{1}{z}\left[\left(\widehat{F}^{(+)}\right)^{-1}+\left(\frac{1}{z}-1\right)\left(\widehat{F}^{(2)}\right)^{-1}+\right. \\
& \left.\left(\widehat{F}^{(+)}\right)^{-1}\left\{\widehat{F}^{(2)} \cdot\left(\widehat{F}^{(-)}\right)^{-1}-\widehat{1}\right\} \Theta(\vec{q})\right]^{-1},
\end{aligned}
$$

where $\widehat{F}^{(+)}=\widehat{F}^{(2)}+\widehat{F}^{(11)}, \widehat{F}^{(-)}=\widehat{F}^{(2)}-\widehat{F}^{(11)}$.

\section{The Blume-Emery-Griffiths model}

Let us consider the reference Blume-Emery-Griffiths model $(\eta=2)$, which is described by the Hamiltonian

$$
{ }^{k} \mathcal{H}\left(\{æ\},\left\{æ^{\prime}\right\}\right)=\beta \sum_{i=1}^{N}\left[æ_{i} S_{i}+æ_{i}^{\prime} S_{i}^{2}\right]+\frac{1}{2} \beta \sum_{i, \delta}\left[K S_{i} S_{i+\delta}+K^{\prime} S_{i}^{2} S_{i+\delta}^{2}\right] .
$$

Here $æ_{i}=\Gamma_{i}+\sum_{j=1}^{N} J_{i j}\left\langle S_{j}\right\rangle, \quad æ_{i}^{\prime}=D_{i}+\sum_{j=1}^{N} J_{i j}^{\prime}\left\langle S_{j}^{2}\right\rangle, \Gamma_{i}$ is an external field. $D_{i}$ stands for single-ion anisotropy. Let us note that the factor $\beta=\left(k_{B} T\right)^{-1}$ is written explicitly hereafter.

On the basis of $(3.3),(3.7)$ and $(3.9),(3.10)$ we calculate the singleparticle and two-particle $F$-functions

$$
\begin{aligned}
& F_{1}\left(T, \tilde{\mathfrak{x}}_{1}, \tilde{\mathfrak{x}}_{1}^{\prime}\right)=\ln \left(2 \exp \left(4 \beta \tilde{\mathfrak{x}}_{1}^{\prime}\right) \cdot \operatorname{ch}\left(2 \beta \tilde{æ}_{1}\right)+1\right),
\end{aligned}
$$

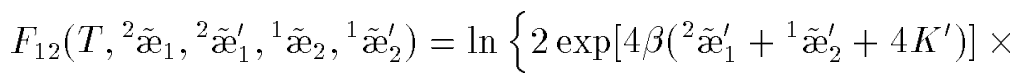

$$
\begin{aligned}
& \left(\exp (4 \beta K) \cdot \operatorname{ch}\left[2 \beta\left({ }^{2} \tilde{\mathfrak{x}}_{1}+{ }^{1} \tilde{\mathfrak{x}}_{2}\right)\right]+\exp (-4 \beta K) \cdot \operatorname{ch}\left[2 \beta\left({ }^{2} \tilde{\mathfrak{x}}_{1}-{ }^{1} \tilde{\mathfrak{x}}_{2}\right)\right]\right)+ \\
& \left.2 \exp \left(4 \beta^{2} \tilde{\mathfrak{X}}_{1}^{\prime}\right) \cdot \operatorname{ch}\left(2 \beta^{2} \tilde{\mathfrak{X}}_{1}\right)+2 \exp \left(4 \beta^{1} \tilde{\mathfrak{x}}_{2}^{\prime}\right) \cdot \operatorname{ch}\left(2 \beta^{1} \tilde{\mathfrak{X}}_{2}\right)+1\right\} \text {. }
\end{aligned}
$$


In uniform fields, that is, when the relations

$$
\begin{aligned}
& \tilde{\mathfrak{X}}_{1}=\tilde{\mathfrak{X}}=æ 2+z \cdot \varphi ; \quad{ }^{r} \tilde{\mathfrak{X}}_{1}=\tilde{\tilde{\dddot{x}}}=æ 2+(z-1) \varphi ; \\
& \tilde{æ}_{1}^{\prime}=\tilde{\dddot{x}}^{\prime}=\mathfrak{x}^{\prime}+z \cdot \varphi^{\prime} ; \quad{ }^{r} \tilde{\dddot{x}}_{1}^{\prime}=\tilde{\tilde{x}}^{\prime}=\mathfrak{x}^{\prime}+(z-1) \varphi^{\prime}
\end{aligned}
$$

hold, we have

$$
\begin{aligned}
& \frac{1}{N}{ }^{k} \mathcal{F}\left(T, \Gamma, D, \varphi, \varphi^{\prime},\langle S\rangle,\left\langle S^{2}\right\rangle\right)=(1-z) F_{1}\left(T, \tilde{x}, \tilde{x}^{\prime}\right)+\frac{z}{2} F_{12}\left(T, \tilde{\tilde{x}}, \tilde{\tilde{x}}^{\prime}\right) ; \\
& F_{1}\left(T, \tilde{x}, \tilde{x}^{\prime}\right)=\ln Z_{1}\left(T, \tilde{x}, \tilde{x}^{\prime}\right) ; \quad F_{12}\left(T, \tilde{\tilde{x}}, \tilde{\tilde{x}}^{\prime}\right)=\ln Z_{12}\left(T, \tilde{\tilde{x}}, \tilde{\tilde{x}}^{\prime}\right) ; \\
& Z_{1}\left(T, \tilde{x}, \tilde{x}^{\prime}\right)=2 \mathrm{e}^{4 \beta \tilde{\dddot{x}}^{\prime}} \cdot \operatorname{ch}(2 \beta \tilde{x})+1 ; \\
& Z_{12}\left(T, \tilde{\tilde{x}}, \tilde{\tilde{x}}^{\prime}\right)= 2 \mathrm{e}^{8 \beta\left(\tilde{\tilde{x}}^{\prime}+2 K^{\prime}\right)}\left(\mathrm{e}^{4 \beta K} \cdot \operatorname{ch}(4 \beta \tilde{\tilde{x}})+\mathrm{e}^{-4 \beta K}\right)+ \\
& 4 \mathrm{e}^{4 \beta \tilde{\tilde{x}}^{\prime}} \cdot \operatorname{ch}(2 \beta \tilde{\tilde{x}})+1 .
\end{aligned}
$$

Let us note that if $J=J^{\prime}=0$ the dependence ${ }^{k} \mathcal{F}={ }^{k} \mathcal{F}\left(T, \Gamma, D, \varphi, \varphi^{\prime}\right)$ takes place. To obtain an explicit system of equations for the cluster fields $\varphi$, $\varphi^{\prime}$, single-site CFs $\langle S\rangle,\left\langle S^{2}\right\rangle$ and expressions for pair CFs, we need to know intracluster CFs $F_{1}\left(\begin{array}{c}1 \\ n\end{array}\right), F_{1}\left(\begin{array}{c}2 \\ n\end{array}\right), F_{1}\left(\begin{array}{c}1 \\ n\end{array}\left|\begin{array}{l}1 \\ m\end{array}\right|\right), F_{1 r}\left(\begin{array}{c}1 \\ n\end{array}\right), F_{1 r}\left(\begin{array}{c}2 \\ n\end{array} \mid\right), F_{1 r}\left(\begin{array}{c}1 \\ n\end{array}\left|\begin{array}{l}1 \\ m\end{array}\right|\right)$. Differentiating $F$-functions with respect to relevant variables $(n, m=1$ and $n, m=2$ correspond to differentiation to single-particle $F_{1}$-function with respect to $\tilde{æ}_{1}$ and $\tilde{æ}_{1}^{\prime}$ (see (3.15)) and of two-particle $F_{12}$-function with respect to ${ }^{2} \tilde{\mathfrak{x}}_{1}$ and ${ }^{2} \tilde{\mathfrak{x}}_{1}^{\prime}$, or ${ }^{1} \tilde{\mathfrak{x}}_{2}$ and ${ }^{1} \tilde{\mathfrak{x}}_{2}^{\prime}$ (see (3.16))) and going to the uniform fields case, we obtain

$$
\begin{aligned}
& F_{1}\left(\begin{array}{l}
1 \\
1
\end{array}\right)=4 \mathrm{e}^{4 \beta \tilde{x}^{\prime}} \cdot \operatorname{sh}(2 \beta \tilde{x}) \cdot Z_{1}^{-1} ; \\
& F_{1}\left(\begin{array}{l}
1 \\
2
\end{array}\right)=8 \mathrm{e}^{4 \beta \tilde{x}^{\prime}} \cdot \operatorname{ch}(2 \beta \tilde{x}) \cdot Z_{1}^{-1} ;
\end{aligned}
$$

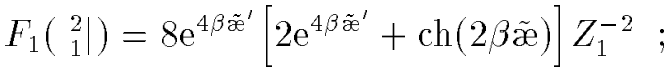

$$
\begin{aligned}
& F_{1}\left(\begin{array}{l}
2 \\
2
\end{array}\right)=32 \mathrm{e}^{4 \beta \tilde{x}^{\prime}} \cdot \operatorname{ch}(2 \beta \tilde{x}) \cdot Z_{1}^{-2} ; \\
& F_{1}\left(\begin{array}{l}
1 \\
1
\end{array} \mid \begin{array}{l}
1 \\
2
\end{array}\right)=F_{1}\left(\begin{array}{l}
1 \\
2
\end{array} \mid \begin{array}{l}
1 \\
1
\end{array}\right)=16 \mathrm{e}^{4 \beta \tilde{\varkappa}^{\prime}} \cdot \operatorname{sh}(2 \beta \tilde{x}) \cdot Z_{1}^{-2} ;
\end{aligned}
$$

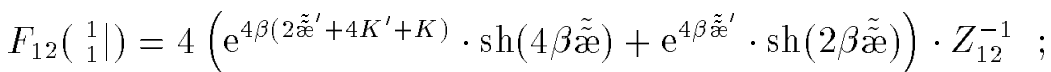

$$
\begin{aligned}
& F_{12}\left(\begin{array}{l}
1 \\
2
\end{array}\right)=8\left\{\mathrm{e}^{8 \beta\left(\tilde{\tilde{x}}^{\prime}+2 K^{\prime}\right)}\left[\mathrm{e}^{4 \beta K} \cdot \operatorname{ch}(4 \beta \tilde{\tilde{x}})+\mathrm{e}^{-4 \beta K}\right]+\right. \\
& \left.\mathrm{e}^{4 \beta \tilde{\tilde{\ddot{x}}}^{\prime}} \cdot \operatorname{ch}(2 \beta \tilde{\tilde{x}})\right\} \cdot Z_{12}^{-1} \quad ;
\end{aligned}
$$

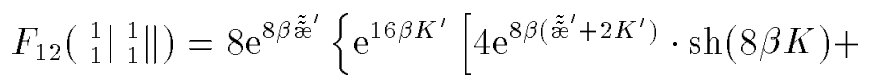

$$
\begin{aligned}
& 8 \mathrm{e}^{4 \beta \tilde{\tilde{x}}^{\prime}} \cdot \operatorname{sh}(4 \beta K) \cdot \operatorname{ch}(2 \beta \tilde{\tilde{x}})+ \\
& \left.\left.\mathrm{e}^{4 \beta K} \cdot \operatorname{ch}(4 \beta \tilde{\tilde{x}})-\mathrm{e}^{-4 \beta K}\right]-2 \operatorname{sh}^{2}(2 \beta \tilde{\tilde{x}})\right\} \cdot Z_{12}^{-2} ;
\end{aligned}
$$

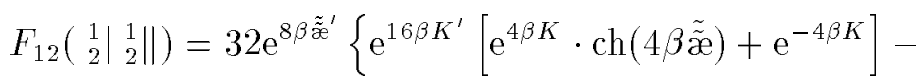

$$
\begin{aligned}
& \left.2 \operatorname{ch}^{2}(2 \beta \tilde{\tilde{x}})\right\} \cdot Z_{12}^{-2} \\
& F_{12}\left(\begin{array}{l}
1 \\
1
\end{array}\left|\begin{array}{l}
1 \\
2
\end{array}\right|\right)=F_{12}\left(\begin{array}{l}
1 \\
2
\end{array}\left|\begin{array}{l}
1 \\
1
\end{array}\right|\right)=16 \mathrm{e}^{8 \beta \tilde{\tilde{\tilde{x}}}^{\prime}}\left\{4 \mathrm{e}^{4 \beta\left(\tilde{\tilde{x}}^{\prime}+4 K^{\prime}\right)} \cdot \operatorname{sh}(2 \beta \tilde{\tilde{\tilde{x}}}) \cdot \operatorname{sh}(4 \beta K)+\right. \\
& \left.\mathrm{e}^{4 \beta\left(4 K^{\prime}+K\right)} \cdot \operatorname{sh}(4 \beta \tilde{\tilde{x}})-2 \operatorname{sh}(2 \beta \tilde{\tilde{x}}) \cdot \operatorname{ch}(2 \beta \tilde{\tilde{x}})\right\} \cdot Z_{12}^{-2} .
\end{aligned}
$$


On the basis of $(3.18),(3.19)$ and $(4.7),(4.8)$ we can write an explicit system of equations for $\varphi, \varphi^{\prime},\langle S\rangle,\left\langle S^{2}\right\rangle$ in the uniform fields case:

$$
\begin{aligned}
& \langle S\rangle=\frac{4 \mathrm{e}^{4 \beta \tilde{æ}^{\prime}} \cdot \operatorname{sh}(2 \beta \tilde{æ})}{Z_{1}\left(T, \tilde{æ}, \tilde{æ}^{\prime}\right)} ; \quad\left\langle S^{2}\right\rangle=\frac{8 \mathrm{e}^{4 \beta \tilde{æ}^{\prime}} \cdot \operatorname{ch}(2 \beta \tilde{æ})}{Z_{1}\left(T, \tilde{æ}, \tilde{æ}^{\prime}\right)} ;
\end{aligned}
$$

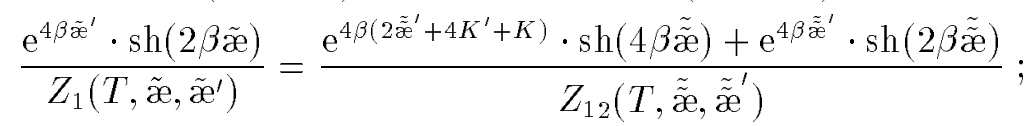

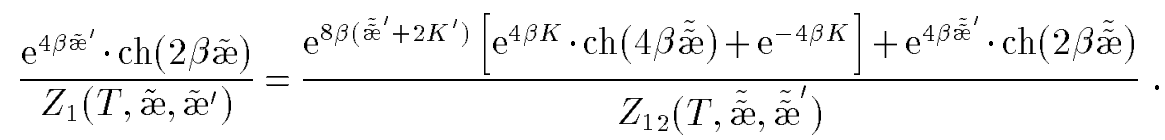

Let us note, that when long-range interactions are absent $\left(J=J^{\prime}=0\right)$ we obtain the system of equations (4.10) for the cluster fields $\varphi, \varphi^{\prime}$ and expressions (4.9) for $\langle S\rangle,\left\langle S^{2}\right\rangle$ (expressions (4.9) and the system of equations (4.10) are in agreement with the results of constant coupling approximation $[17])$.

On the basis of $(3.42)(\eta=2)$, considering the fact that $F_{1}\left(\begin{array}{ll}1 & 1 \\ 1 & 2\end{array} \mid\right)=$ $F_{1}\left(\begin{array}{c}1 \\ 2\end{array} \mid \begin{array}{l}1 \\ 1\end{array}\right), F_{12}\left(\begin{array}{c}1 \\ 1\end{array}\left|\begin{array}{l}1 \\ 2\end{array}\right|\right)=F_{12}\left(\begin{array}{c}1 \\ 2\end{array}\left|\begin{array}{l}1 \\ 1\end{array}\right|\right)$, we can obtain expressions for pair CFs of the reference $\mathrm{BEG}$ model.

$$
k \widehat{b}(\vec{q})=\left(\begin{array}{cc}
{ }^{k}\langle S S\rangle_{\vec{q}}^{c} & { }^{k}\left\langle S S^{2}\right\rangle_{\vec{q}}^{c} \\
{ }^{k}\left\langle S^{2} S\right\rangle_{\vec{q}}^{c} & { }^{k}\left\langle S^{2} S^{2}\right\rangle_{\vec{q}}^{c}
\end{array}\right)=\frac{d^{+} d^{-} d^{(2)}}{z\left(\tilde{b}_{1} \tilde{b}_{3}-\left(\tilde{b}_{2}\right)^{2}\right)} \cdot\left(\begin{array}{cc}
\tilde{b}_{1} & \tilde{b}_{2} \\
\tilde{b}_{2} & \tilde{b}_{3}
\end{array}\right)
$$

where

$$
\begin{aligned}
& \tilde{b}_{\alpha}=d^{(-)}\left\{d^{(2)} F_{\alpha}^{(+)}+\left(\frac{1}{z}-1\right) d^{(+)} F_{\alpha}^{(2)}\right\}- \\
& d^{(2)}\left\{F_{\alpha}^{(+)}\left(d^{(-)}+F_{2}^{(-)} F_{2}^{(2)}\right)-B_{\alpha}\right\} \Theta(\vec{q}), \quad(\alpha=1,2,3) \text {; } \\
& \widehat{F}^{(\xi)}=\left(\begin{array}{ll}
F_{1}^{(\xi)} & F_{2}^{(\xi)} \\
F_{2}^{(\xi)} & F_{3}^{(\xi)}
\end{array}\right), \quad(\xi=+,-, 2) \quad ; \quad \widehat{F}^{( \pm)}=\widehat{F}^{(2)} \pm \widehat{F}^{(11)} ; \\
& d^{(-)}=\operatorname{det}\left|\widehat{F}^{(-)}\right| ; \quad d^{(+)}=\operatorname{det}\left|\widehat{F}^{(+)}\right| ; \quad d^{(2)}=\operatorname{det}\left|\widehat{F}^{(2)}\right| ;
\end{aligned}
$$

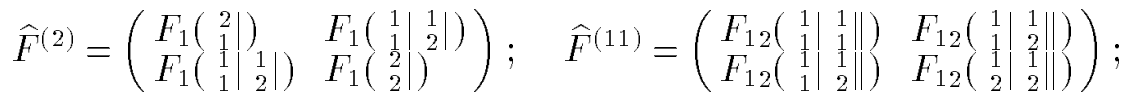

$$
\begin{aligned}
& B_{1}=F_{1}^{(+)} F_{1}^{(-)} F_{3}^{(2)}+F_{2}^{(+)} F_{2}^{(-)} F_{1}^{(2)}-F_{2}^{(+)} F_{1}^{(-)} F_{2}^{(2)} \text {; } \\
& B_{2}=F_{2}^{(+)} F_{1}^{(-)} F_{3}^{(2)}+F_{3}^{(+)} F_{2}^{(-)} F_{1}^{(2)}-F_{3}^{(+)} F_{1}^{(-)} F_{2}^{(2)} \text {; } \\
& B_{3}=F_{3}^{(+)} F_{3}^{(-)} F_{1}^{(2)}+F_{2}^{(+)} F_{2}^{(-)} F_{3}^{(2)}-F_{2}^{(+)} F_{3}^{(-)} F_{2}^{(2)} \text {. }
\end{aligned}
$$

\section{Numerical analysis results}

In this section we discuss the results of numerical calculations $(\Gamma=0)$ of thermodynamic characteristics and pair correlation functions (at $\vec{q}=0$ ) of BEG model on a simple cubic lattice $(z=6)$.

Here we use the following notations for the relative quantities:

$$
\begin{aligned}
& t=\frac{3}{8} \frac{k_{B} T}{z K}, \quad d=\frac{\mathfrak{x}^{\prime}}{K}, \quad k^{\prime}=\frac{4 K^{\prime}}{K}, \quad m=\frac{1}{2}\langle S\rangle, \quad q=\frac{1}{4}\left\langle S^{2}\right\rangle, \\
& G_{11}=\frac{1}{4}\langle S S\rangle_{\vec{q}=0}^{c}, G_{12}=\frac{1}{8}\left\langle S S^{2}\right\rangle_{\vec{q}=0}^{c}, \quad G_{11}=\frac{1}{16}\left\langle S^{2} S^{2}\right\rangle_{\vec{q}=0}^{c}
\end{aligned}
$$


and the terminology of [17]:

$\mathbf{F}$ - the ferromagnetic phase $\left(m \neq 0, q \neq \frac{2}{3}\right)$,

$\mathbf{P}$ - the paramagnetic phase $\left(m=0, q \neq \frac{2}{3}, q(t=\infty)=\frac{2}{3}\right)$,

$\mathbf{Q}$ - the quadrupolar phase $\left(m=0, q \neq \frac{2}{3}\right)$.

In the two-particle cluster approximation the system of equations for $\varphi, \varphi^{\prime}$ (4.10) has several solutions, the number of which depends on values of parameters $d, k^{\prime}$ and temperature. Solution corresponding to $\mathbf{P}$-phase exists at $t \in\left[t_{\mathrm{P}_{1}}, \infty\right]\left(t_{\mathrm{P}_{1}} \geq 0\right.$, its value depends on $\left.d, k^{\prime}\right)$. Solutions corresponding to the $\mathbf{F}$-phase and $\mathbf{Q}$-phase exist at $t \in\left[t_{\mathrm{F}_{1}}, t_{\mathrm{F}_{2}}\right]$ and $t \in\left[t_{\mathrm{Q}_{1}}, t_{\mathrm{Q}_{2}}\right]$, respectively. The values of $t_{\mathrm{F}_{1}}, t_{\mathrm{F}_{2}}$ and $t_{\mathrm{Q}_{1}}, t_{\mathrm{Q}_{2}}$ depend on $d, k^{\prime}$ and are finite.

The projection of the phase diagram on $\left(d, k^{\prime}\right)$ plane at $d<0, k^{\prime}>-0.1$ [17] and $d>0, k^{\prime}>-1-\frac{1}{6} d$ (see figure 1) consists of seven regions:

I - the phase transition $\mathbf{Q} \leftrightarrow \mathbf{P}$ of the first order ( $\mathbf{Q P} 1)$,

II - the PT FP2,

III - the PT FP1,

IV - the PT is absent,

$\mathrm{V}$ - the PTs QF1 and FP2,

VI - the PTs $\mathbf{Q F} 1$ and $\mathbf{F P} 1$,

VII - the PTs FQ1 and QP 1 .

In the present paper we restrict our consideration to the regions $d<0$, $k^{\prime}>-0.1$ and $d>0, k^{\prime}>-1-\frac{1}{6} d$, since for the regions $d>0, k^{\prime}<-1-\frac{1}{6} d$ and $d<0, k^{\prime}<-1$ a two-sublattice model should be considered [18]. Let us note that in the region $d<0,-0.1>k^{\prime}>-1$ in the vicinity of the line $k^{\prime}=-1-\frac{1}{3} d$ the regions with different numbers of various PTs exist. Construction of a projection of a phase diagram on $\left(d, k^{\prime}\right)$ plane and study of thermodymamic properties of the model in these regions are subject of a separate paper.

In figures 2,3 we show the phase diagrams in $(t, d)$ plane for $k^{\prime}=-0.1$, $0.0,1.0,2.0,2.6$ (regions II, III, IV), $k^{\prime}=2.88$ (regions II, III, VII, III, IV), $k^{\prime}=2.95$ (regions II, III, VII, I, IV), $k^{\prime}=3.2$ (regions II, III, VI, I, IV), $k^{\prime}=3.44$ (regions II, V, VI, I, IV), $k^{\prime}=4.0,8.0,20.0$ (regions II, V, I, IV). Hereafter our consideration will imply increasing temperature and decreasing single-ion anisotropy.

Let us briefly consider the pair CFs.

At the transition FP2 (region II and V) the inverse CF $G_{12}^{-1}$ has an infinite discontinuity $\left(G_{12}^{-1}\left(t_{c}-0\right)=0, G_{12}^{-1}\left(t_{c}+0\right)=\infty\right)$, whereas the inverse $\mathrm{CF} G_{11}^{-1}\left(t_{c}\right)=0$ (see figures $4,5,6,9,18,22,25$ ). The inverse $\mathrm{CF} G_{22}^{-1}$ has a finite discontinuity $\left(G_{22}^{-1}\left(t_{c}-0\right)<G_{22}^{-1}\left(t_{c}+0\right)\right)$, and always decreases in the phase $\mathbf{P}$.

In the vicinity of the region $d>0, k^{\prime}<-1-\frac{1}{6} d$ CFs $G_{11}^{-1}, G_{12}^{-1}, G_{22}^{-1}$ are finite and $q \neq 1, m \neq 1$ at $t=0$ (see figures $4(\mathrm{c}$ ), 5(b)). A little bit away from the region $d>0, k^{\prime}<-1-\frac{1}{6} d$ (increasing $d$ or increasing $k^{\prime}$ ) the quantities $q, m, G_{11}^{-1}, G_{12}^{-1}, G_{22}^{-1}$ have bends at low temperature (see figure $4(\mathrm{~b}))$. But a little bit farther from this region $d>0, k^{\prime}<-1-\frac{1}{6} d$ this peculiarity in $\mathbf{F}$-phase disappears.

Let us explore now the temperature behaviour of $G_{22}^{-1}\left(t>t_{c}\right)$ when $k^{\prime}$ increases and $d$ decreases (approaching the region $d>0, k^{\prime}<-1-\frac{1}{6} d$; the region III, the region V). Far from the regions III, V (see figures 4, 5, 6(a), $18(\mathrm{a})) G_{22}^{-1}\left(t>t_{c}\right)$ decreases, and this decrease is not always related to a decrease in $q(t)$. For instance, at small negative $k^{\prime}$ in the vicinity of 


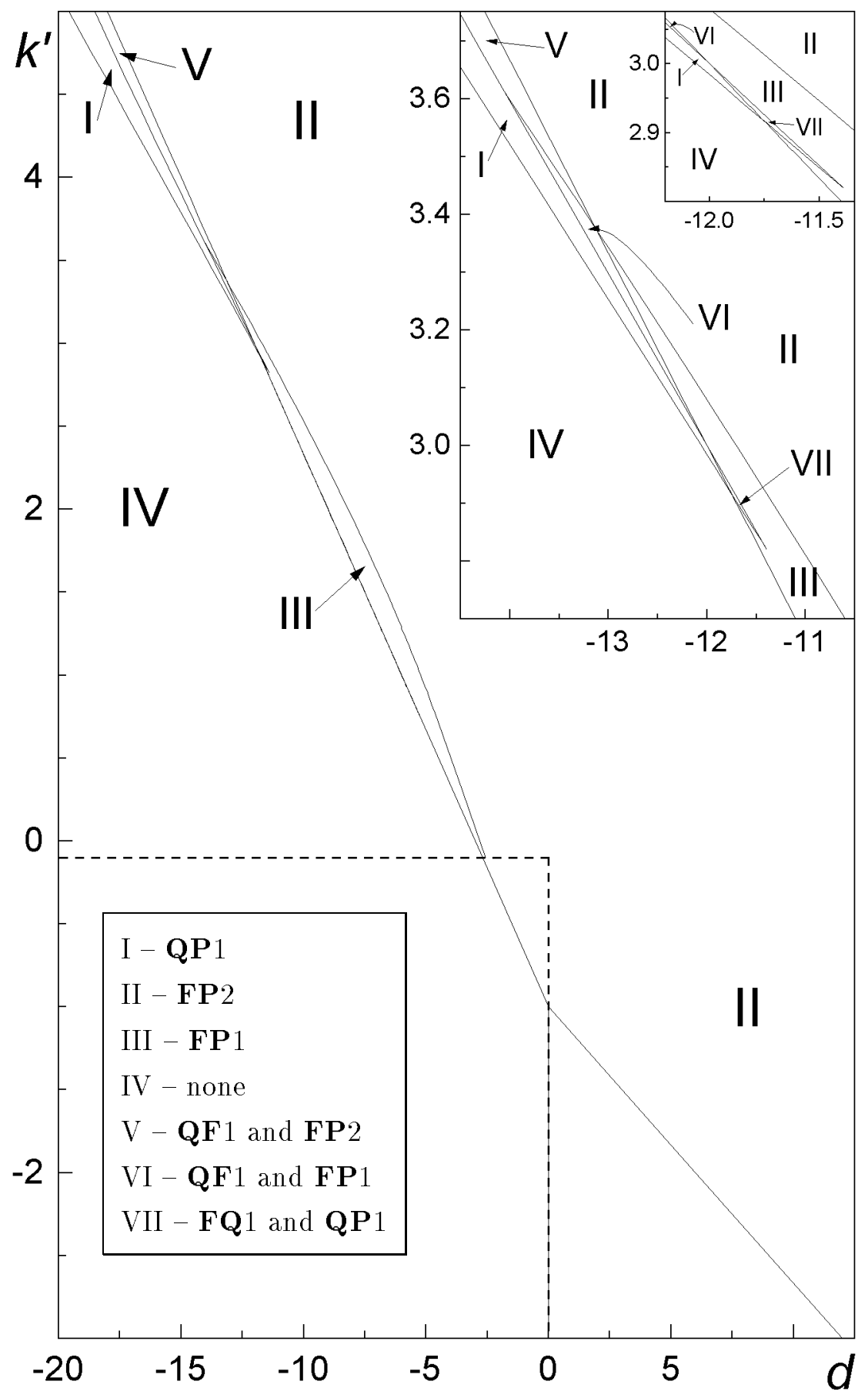

Figure 1. The projection of the phase diagram onto $d-k^{\prime}$ plane. 

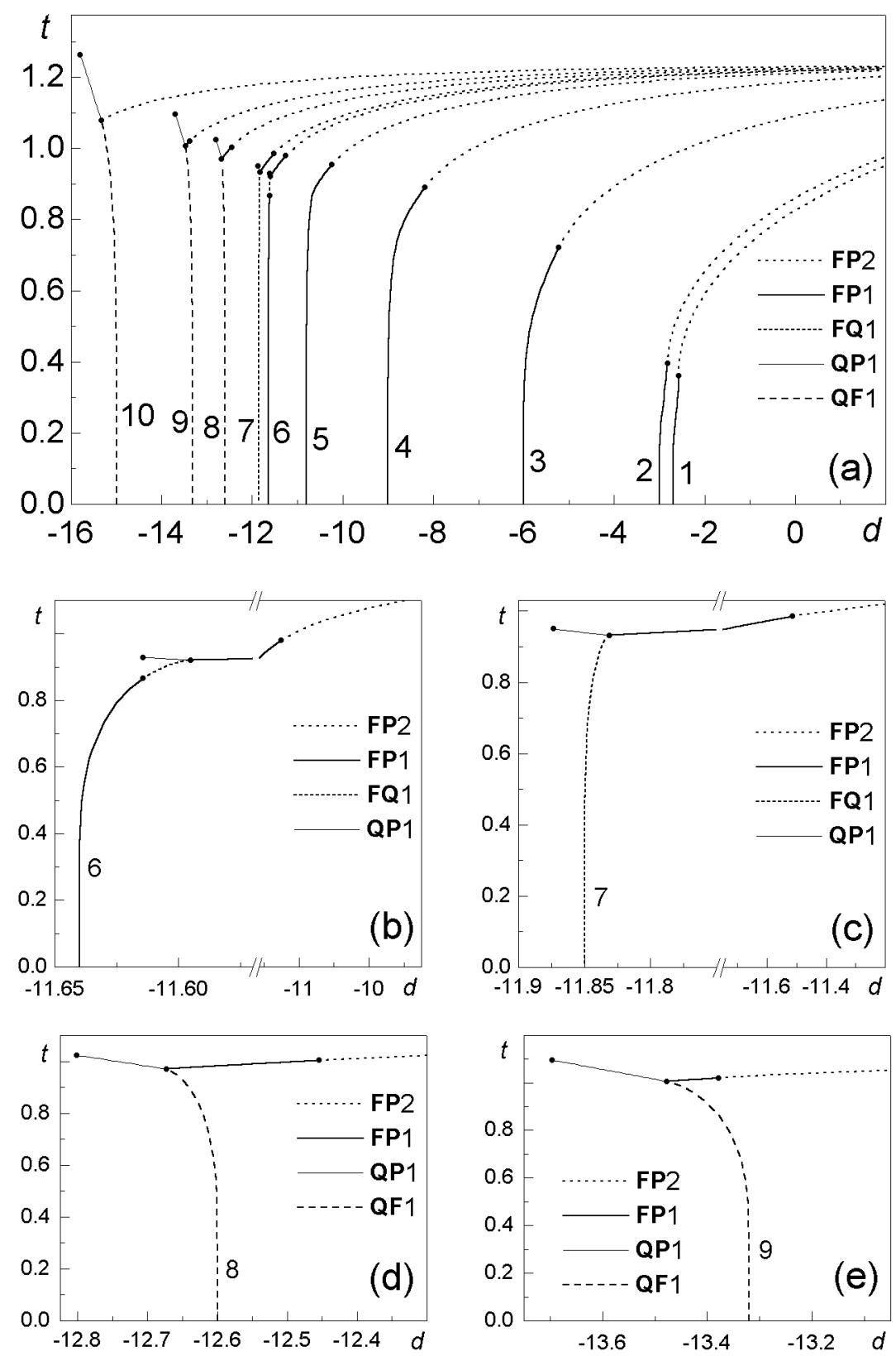

Figure 2. The phase diagram for the several values of $k^{\prime}$ is plotted in $d$ and the reduced temperature plane. $1-k^{\prime}=-0.1$ $2-k^{\prime}=0.0 ; \quad 3-k^{\prime}=1.0 ; \quad 4-k^{\prime}=2.0 ; \quad 5-k^{\prime}=2.6$ $6-k^{\prime}=2.88 ; \quad 7-k^{\prime}=2.95 ; \quad 8-k^{\prime}=3.2 ; \quad 9-k^{\prime}=3.44$ $10-k^{\prime}=4.0$. 


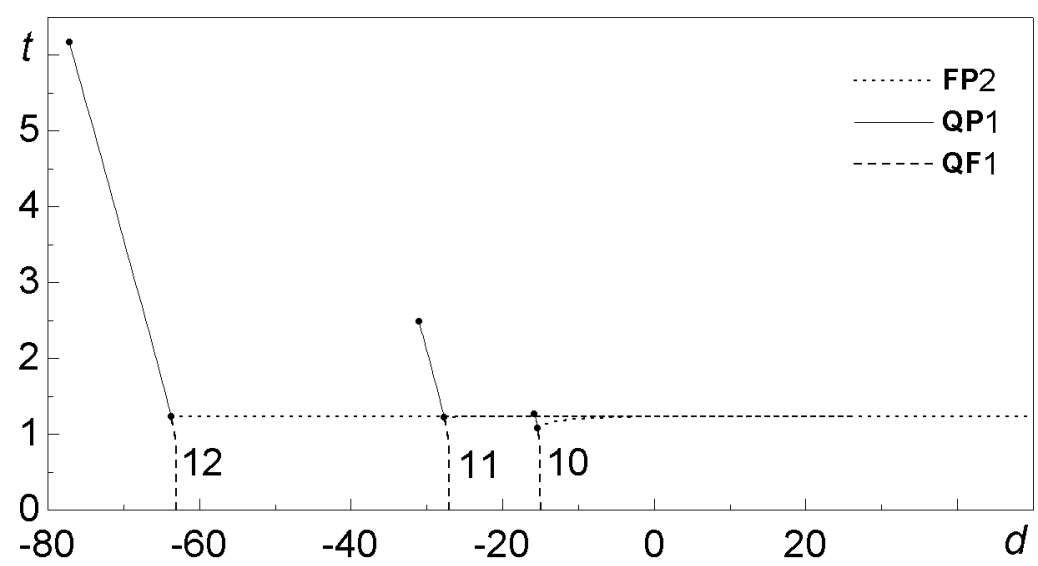

Figure 3. The phase diagram for the several values of $k^{\prime}$ is plotted in $d$ and the reduced temperature plane. $10-k^{\prime}=4.0$; $11-k^{\prime}=8.0 ; \quad 12-k^{\prime}=20.0$.
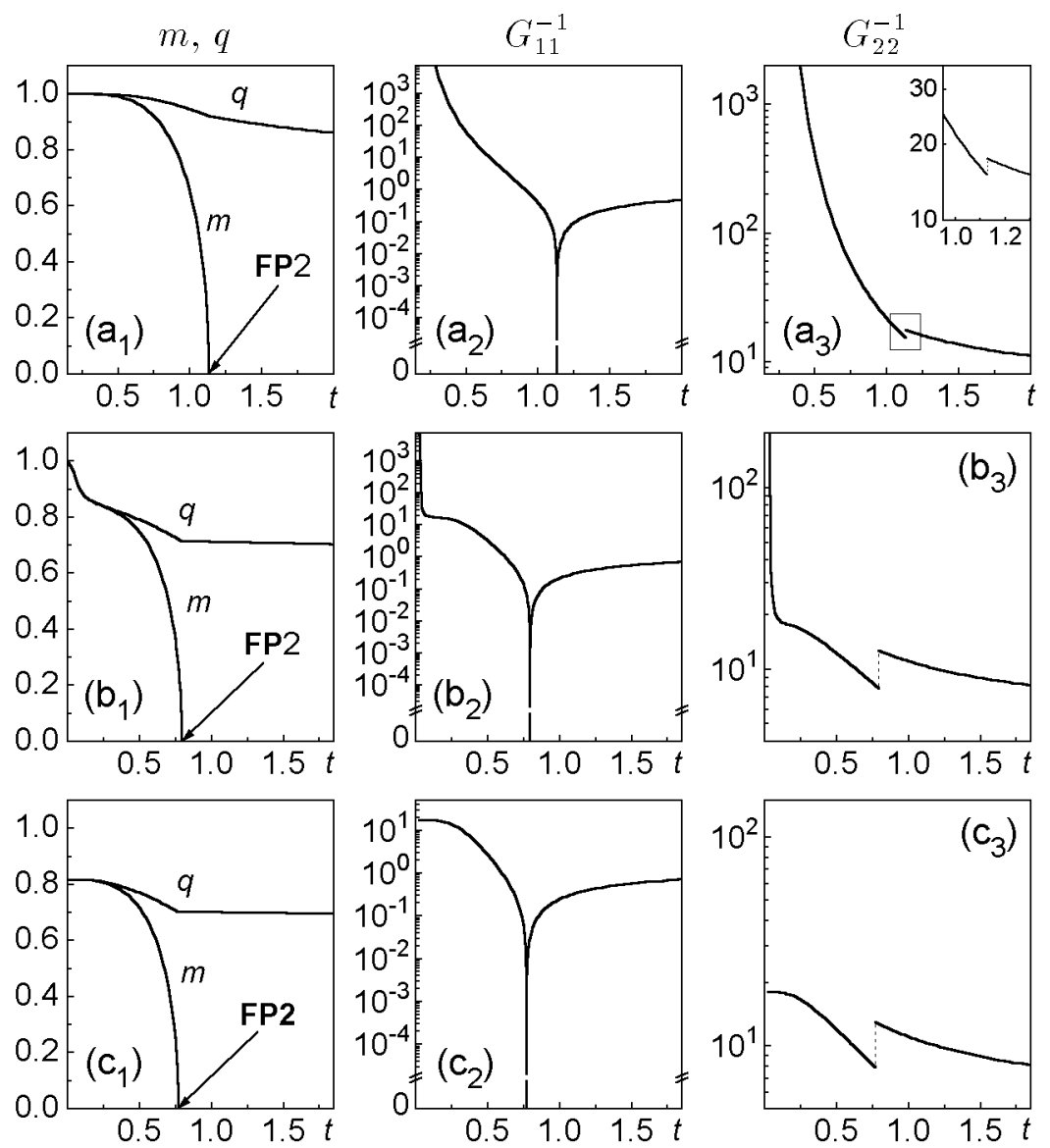

Figure 4. The temperature dependences of $m, q$ and CFs $G_{11}^{-1}(\vec{q}=0)$, $G_{22}^{-1}(\vec{q}=0)$ at $k^{\prime}=-4.0$ for different values of parameter $d:(\mathrm{a})-d=30.0$; (b) $-d=18.5$; (c) $-d=18.0$. 

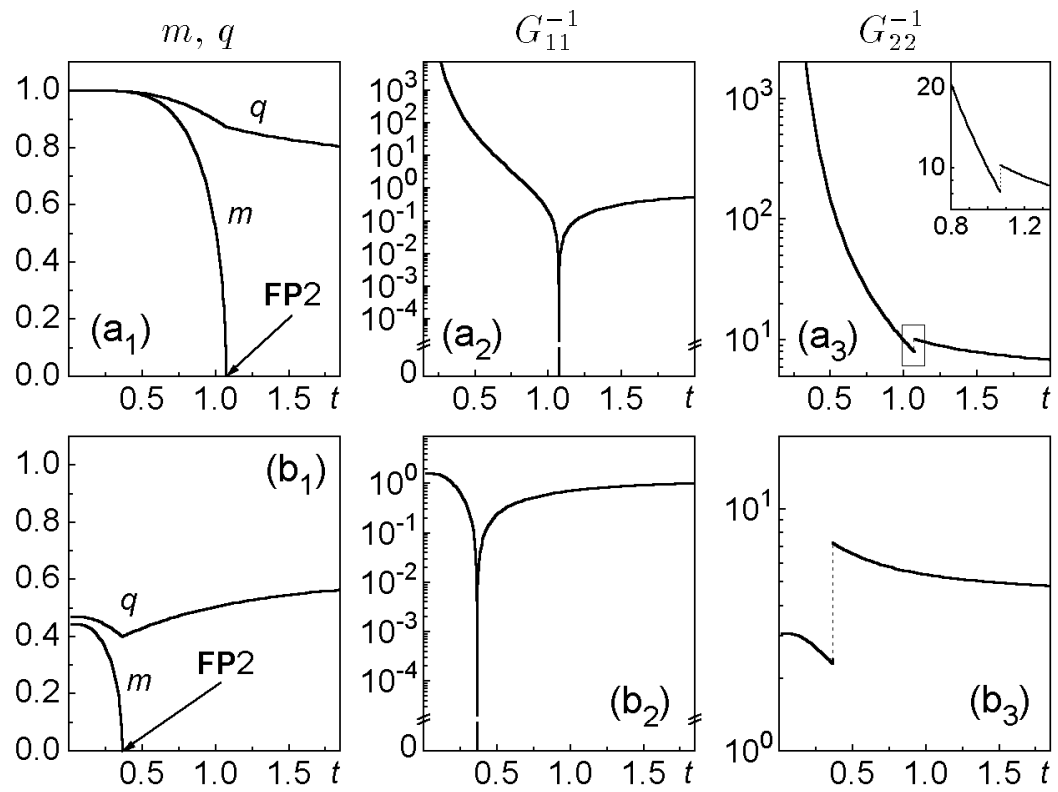

Figure 5. The temperature dependences of $m, q$ and CFs $G_{11}^{-1}(\vec{q}=0)$, $G_{22}^{-1}(\vec{q}=0)$ at $k^{\prime}=-1.0$ for different values of parameter $d:(\mathrm{a})-d=10.0 ;$ (b) $-d=0.0$
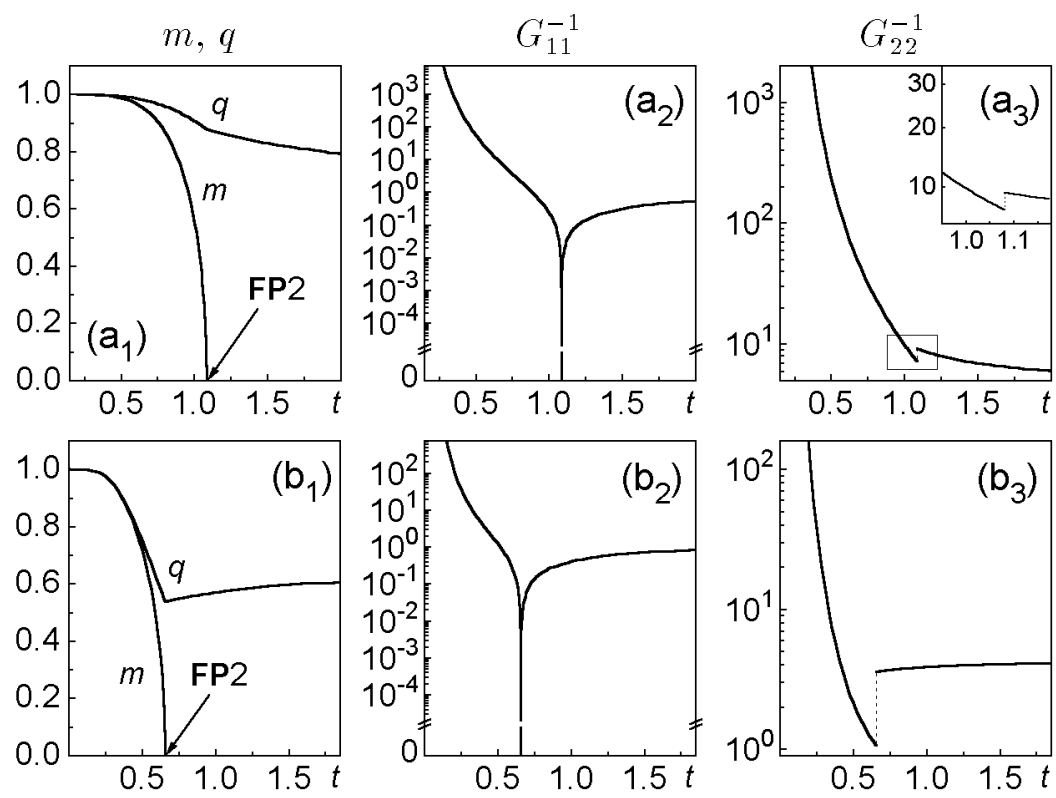

Figure 6. The temperature dependences of $m, q$ and CFs $G_{11}^{-1}(\vec{q}=0)$, $G_{22}^{-1}(\vec{q}=0)$ at $k^{\prime}=0.0$ for different values of parameter $d$ : (a) $-d=5.0 ;$ (b) $-d=-2.0$. 

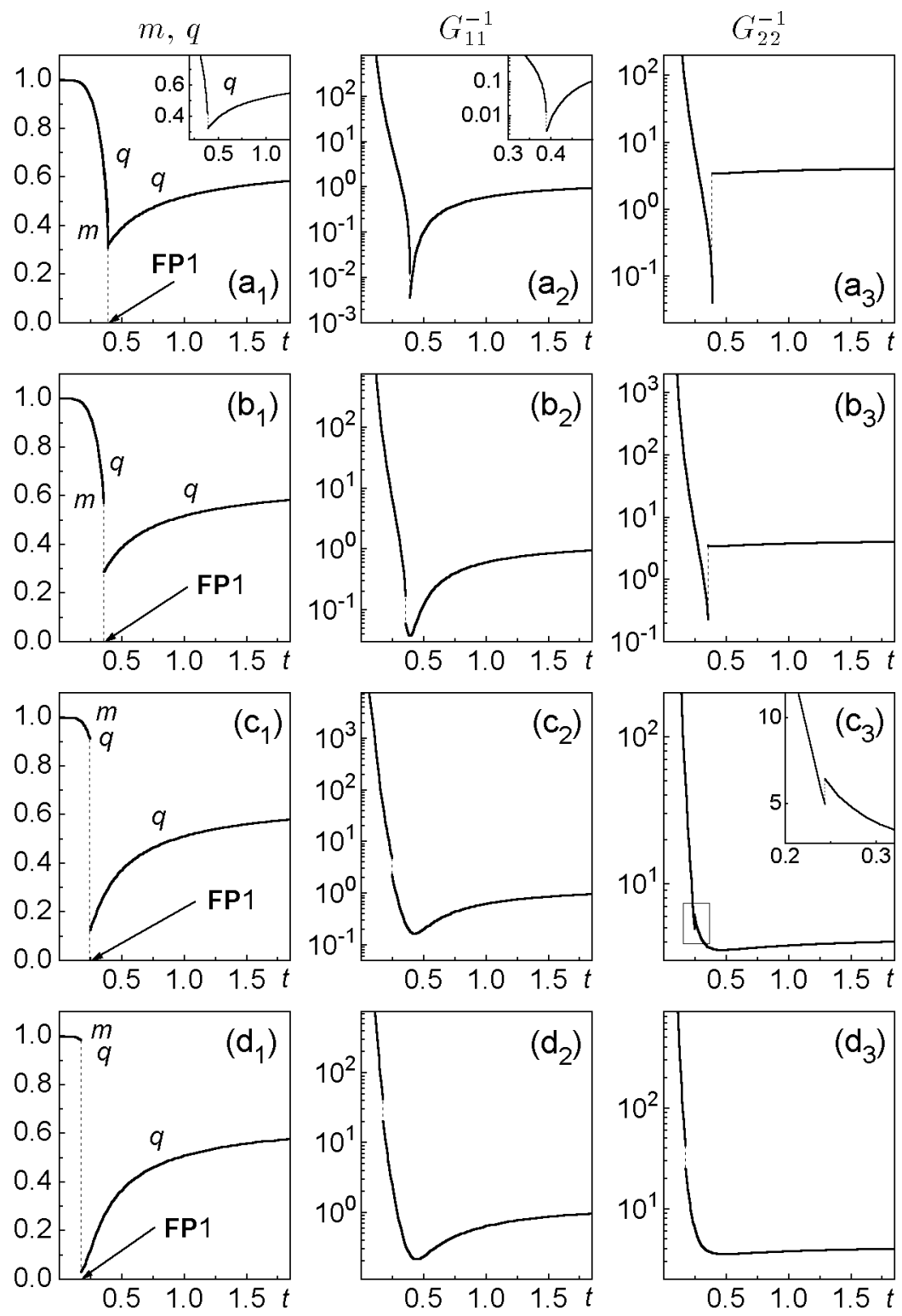

Figure 7. The temperature dependences of $m, q$ and CFs $G_{11}^{-1}(\vec{q}=0)$, $G_{22}^{-1}(\vec{q}=0)$ at $k^{\prime}=0.0$ for different values of parameter $d$ :
(a) $-d=-2.825$;
(b) $-d=-2.85$;
(c) $-d=-2.95$

(d) $-d=-2.99$ 

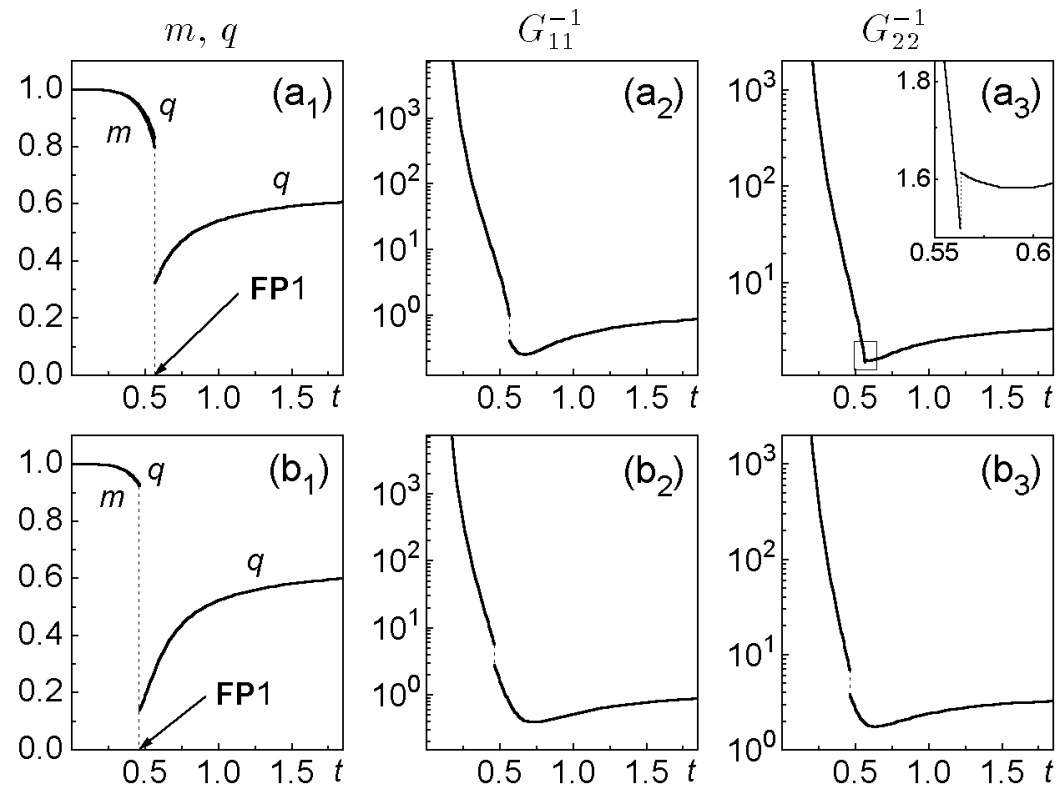

Figure 8. The temperature dependences of $m, q$ and CFs $G_{11}^{-1}(\vec{q}=0)$, $G_{22}^{-1}(\vec{q}=0)$ at $k^{\prime}=1.0$ for different values of parameter $d$ : (a) $-d=-5.725 ; \quad$ (b) $-d=-5.9$.
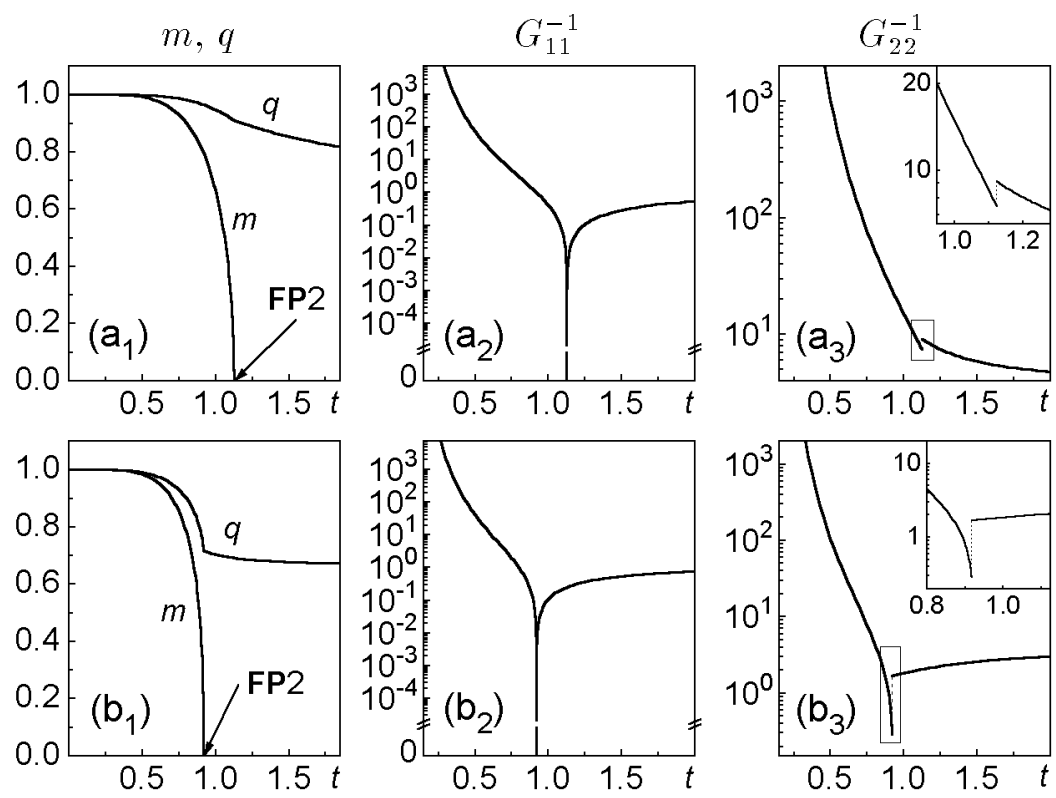

Figure 9. The temperature dependences of $m, q$ and CFs $G_{11}^{-1}(\vec{q}=0)$, $G_{22}^{-1}(\vec{q}=0)$ at $k^{\prime}=2.0$ for different values of parameter $d$ : (a) $-d=-4.0 ; \quad$ (b) $-d=-8.0$. 

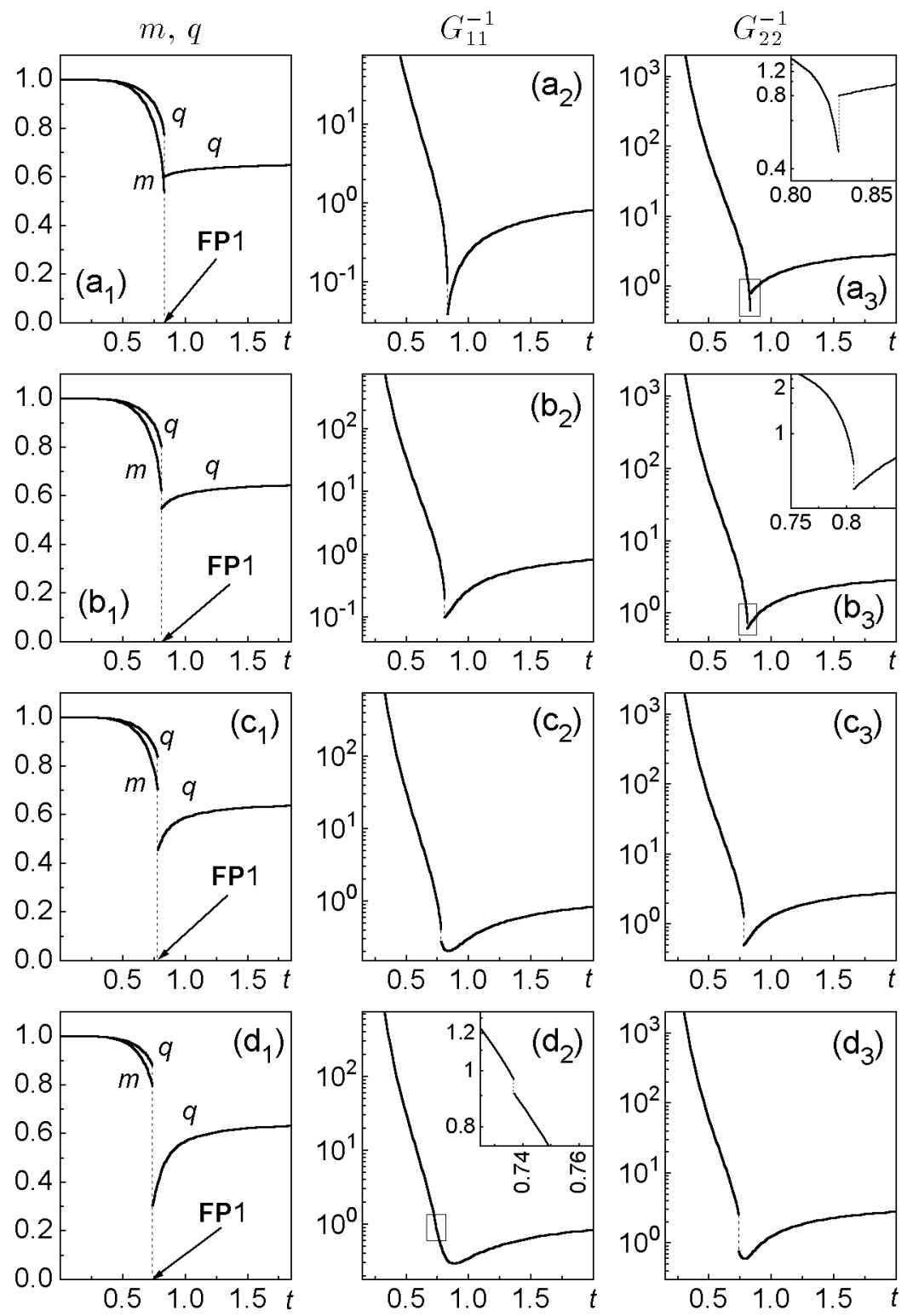

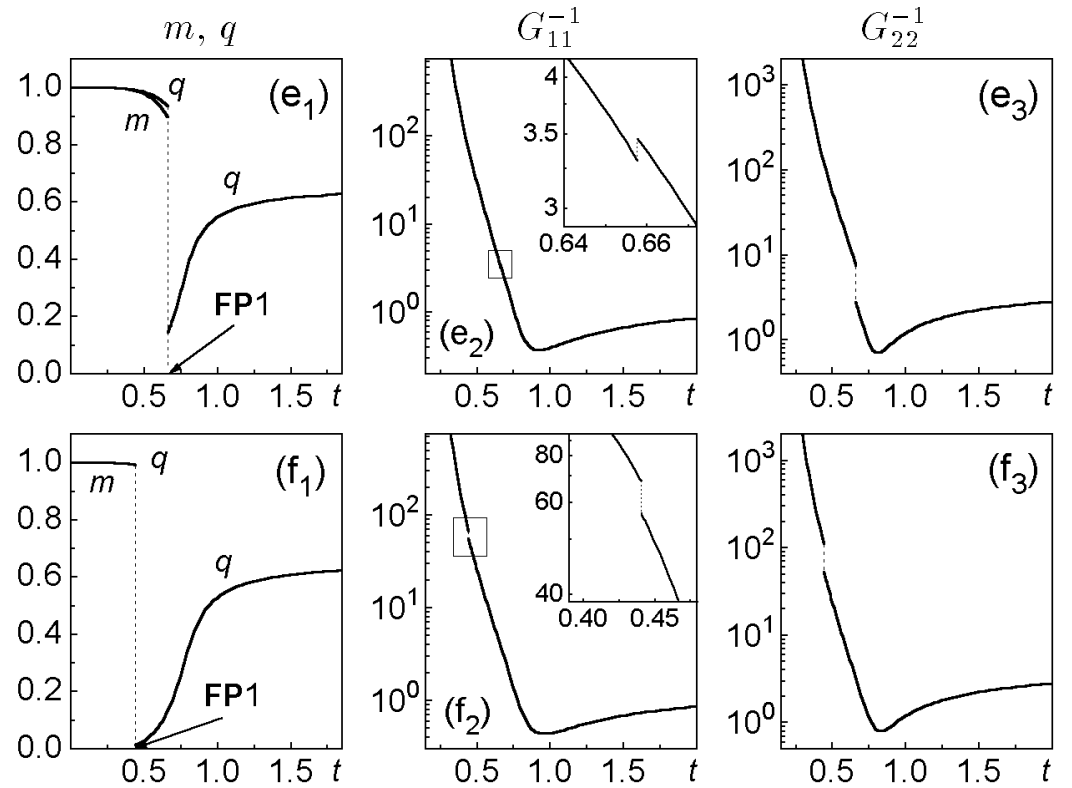

Figure 10. The temperature dependences of $m, q$ and CFs $G_{11}^{-1}(\vec{q}=0)$, $G_{22}^{-1}(\vec{q}=0)$ at $k^{\prime}=2.0$ for different values of parameter $d$ :
(a) $-d=-8.5$;
(b) $-d=-8.6$;
c) $-d=-8.7$;
(d) $-d=-8.8$;
(e) $-d=-8.9$;
(f) $-d=-8.99$.

(Parts (a), (b), (c), (d) of the figure are on previous page.)

the region $d>0, k^{\prime}<-1-\frac{1}{6} d q(t)$ increases, but $G_{22}^{-1}(t)$ decreases (see figure $5(\mathrm{~b}))$. At small positive $k^{\prime}$ with decreasing $d$ (approaching the region III) $G_{22}^{-1}\left(t>t_{c}\right)$ and $q\left(t>t_{c}\right)$ start to increase with temperature (see figure $6(\mathrm{~b})$ ), although at some values of parameters $q$ decreases but $G_{22}^{-1}$ increases (see figure $9(\mathrm{~b})$ ). At larger values of $k^{\prime}$ the decreasing of $d$ (approaching the regions III, V and in the region V) lead to the fact that $G_{22}^{-1}$ has a minimum in the $\mathbf{P}$-phase (see figures 18(b), 22, 25).

At $\mathbf{F P} 1$, the inverse correlation function $G_{12}^{-1}(t)$ has an infinite discontinuity $\left(G_{12}^{-1}\left(t_{c}-0\right)>0, G_{12}^{-1}\left(t_{c}+0\right)=\infty\right)$, whereas CFs $G_{11}^{-1}(t)$ and $G_{22}^{-1}(t)$ have finite discontinuities and decrease in the $\mathbf{F}$-phase. Let us analyse the behaviour of $G_{11}^{-1}(t)$ and $G_{22}^{-1}(t)$ in the vicinity of $\mathbf{F P} 1$ phase transition and in the $\mathbf{P}$-phase in the region III for $k^{\prime}=0.0,1.0,2.0,2.6,2.88,2.95$ when the value of the single-ion anisotropy decreases (moving from the region II towards the region IV for $k^{\prime}=0.0,1.0,2.0,2.6$; moving from the region II towards the region VII for $k^{\prime}=2.88,2.95$; between the regions VII and IV for $k^{\prime}=2.88$ ), in regions III, VI for $k^{\prime}=3.2$ (moving from the region II towards the region I), and in region VI for $k^{\prime}=3.44$ (moving from the region $\mathrm{V}$ towards the region $\mathrm{I})$. In the region III and near to the region II (see figures 7(a), 10(a), 11(a), 12(a), 15(a), 19) $G_{11}^{-1}\left(t_{c}-0\right)>G_{11}^{-1}\left(t_{c}+0\right)$, $G_{22}^{-1}\left(t_{c}-0\right)<G_{22}^{-1}\left(t_{c}+0\right), G_{11}^{-1}\left(t>t_{c}\right)$ and $G_{22}^{-1}\left(t>t_{c}\right)$ increase at all considered values of $k^{\prime}$ except for $k^{\prime}=3.2$ (see figure 19), at which $G_{22}^{-1}(t)$ has a mimimum in the $\mathbf{P}$-phase. Let us consider the case $k^{\prime}=0$. When $d$ decreases, minimums of $G_{11}^{-1}(t)$ (see figure $7(\mathrm{~b})$ ), and, then, of $G_{22}^{-1}(t)$ 

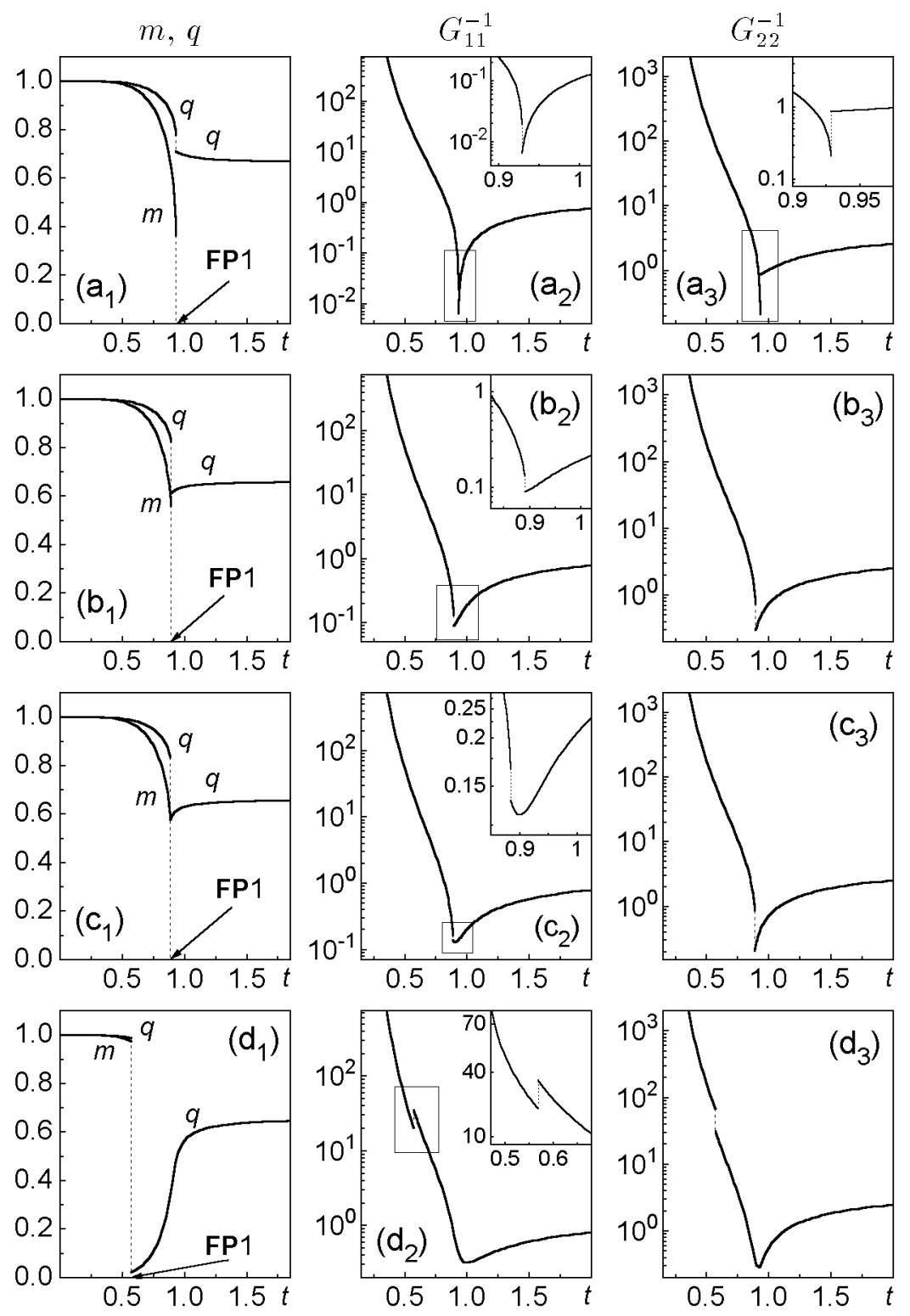

Figure 11. The temperature dependences of $m, q$ and CFs $G_{11}^{-1}(\vec{q}=0)$, $G_{22}^{-1}(\vec{q}=0)$ at $k^{\prime}=2.6$ for different values of parameter $d$ :
(a) $-d=-10.4$
(d) $-d=-10.79$.
(b) $-d=-10.6$;
(c) $-d=-10.625$; 

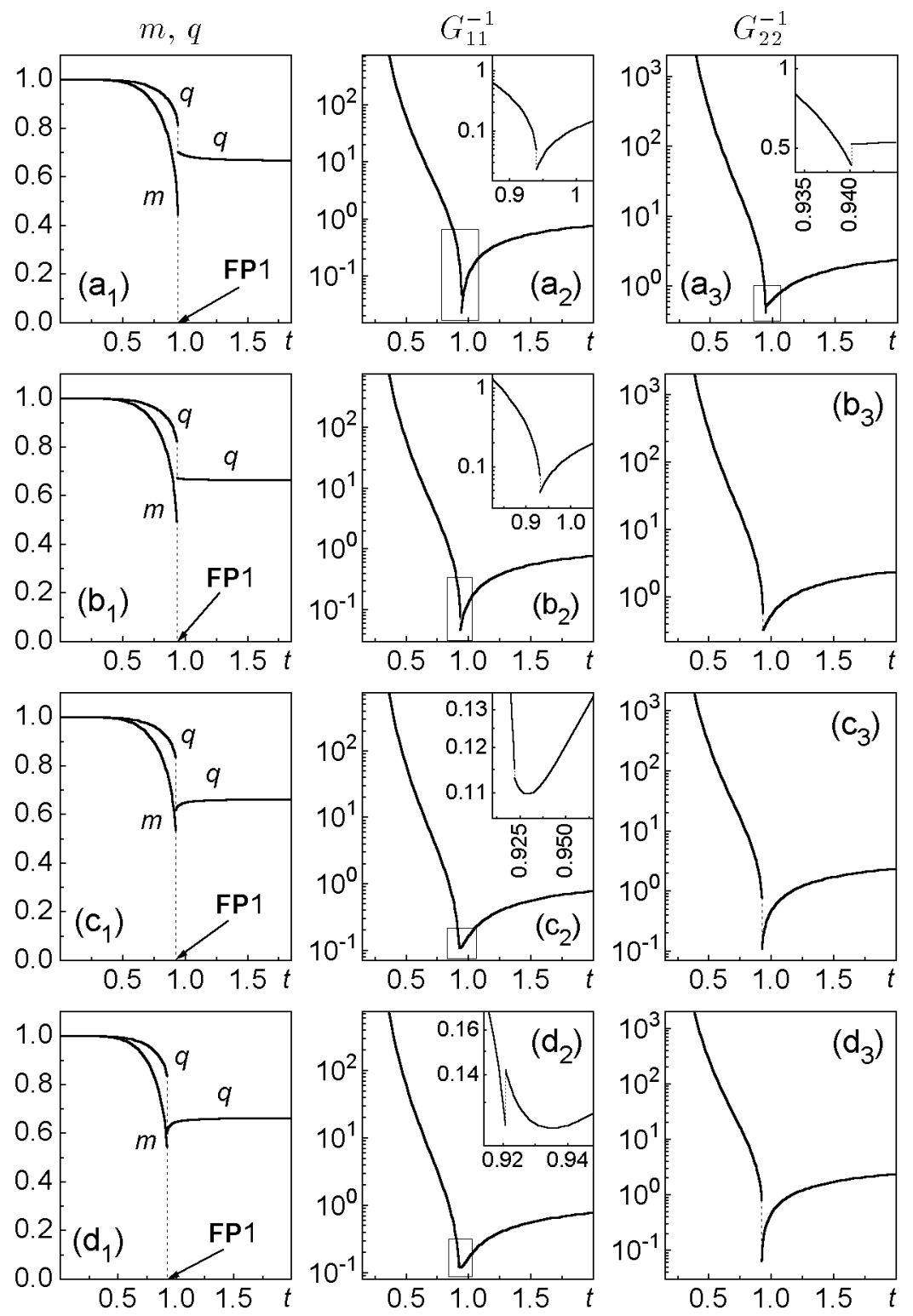

Figure 12. The temperature dependences of $m, q$ and CFs $G_{11}^{-1}(\vec{q}=0)$, $G_{22}^{-1}(\vec{q}=0)$ at $k^{\prime}=2.88$ for different values of parameter $d$ :
(a) $-d=-11.5$;
(d) $-d=-11.595$
(c) $-d=-11.59$; 

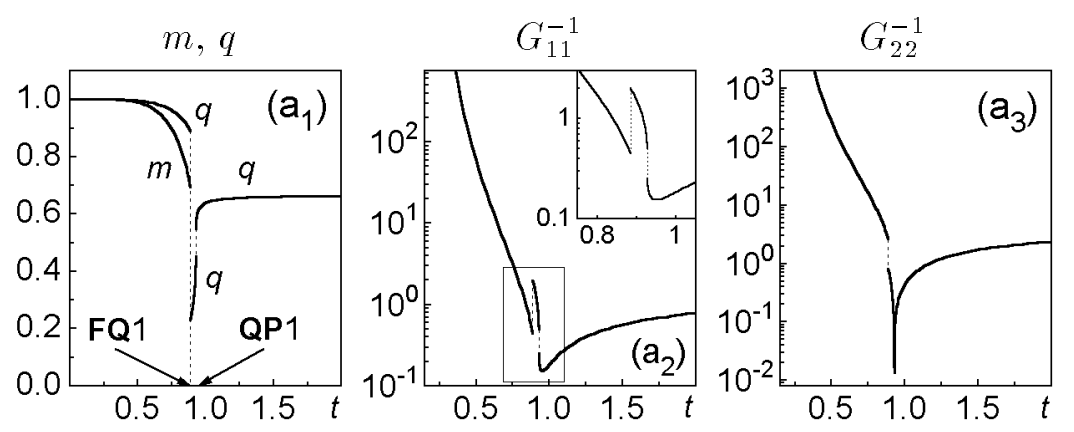

Figure 13. The temperature dependences of $m, q$ and CFs $G_{11}^{-1}(\vec{q}=0)$, $G_{22}^{-1}(\vec{q}=0)$ at $k^{\prime}=2.88$ for $d=-11.61$.
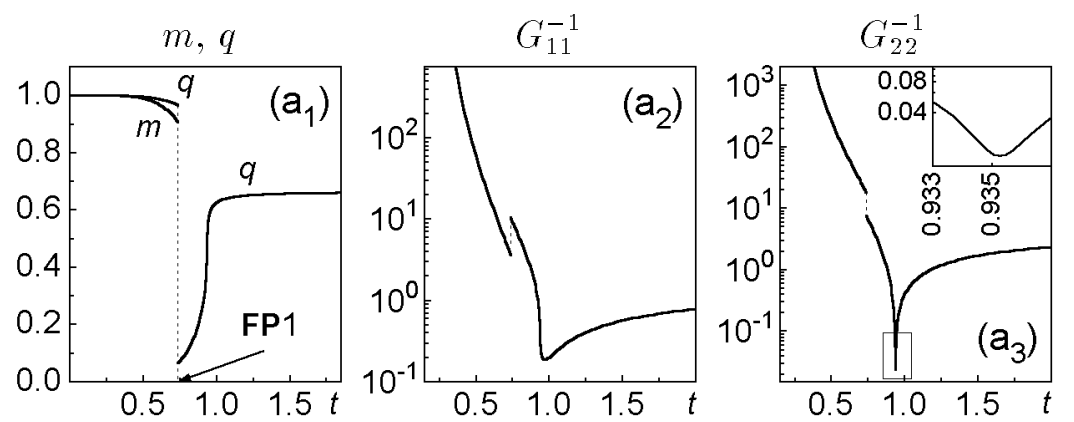

Figure 14. The temperature dependences of $m, q$ and $\mathrm{CFs}_{11}^{-1}(\vec{q}=0)$, $G_{22}^{-1}(\vec{q}=0)$ at $k^{\prime}=2.88$ for $d=-11.63$.
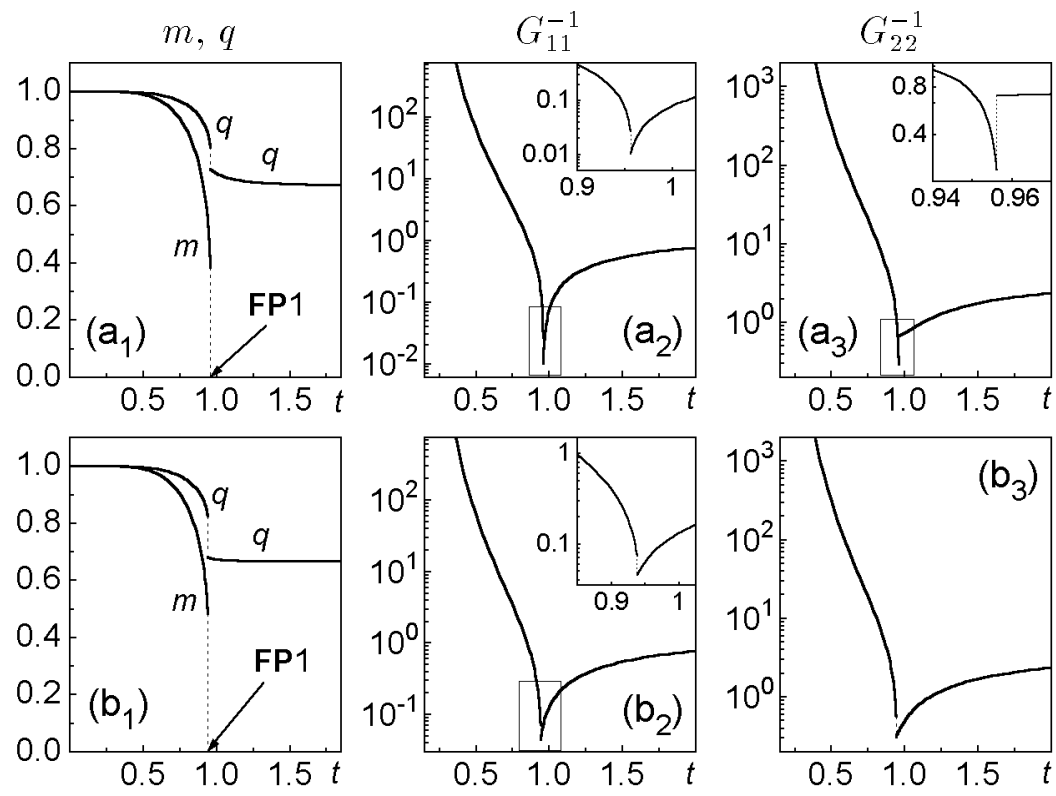

Figure 15. The temperature dependences of $m, q$ and CFs $G_{11}^{-1}(\vec{q}=0)$, $G_{22}^{-1}(\vec{q}=0)$ at $k^{\prime}=2.95$ for different values of parameter $d:($ a) $-d=-11.7 ; \quad$ (b) $-d=-11.8$. 

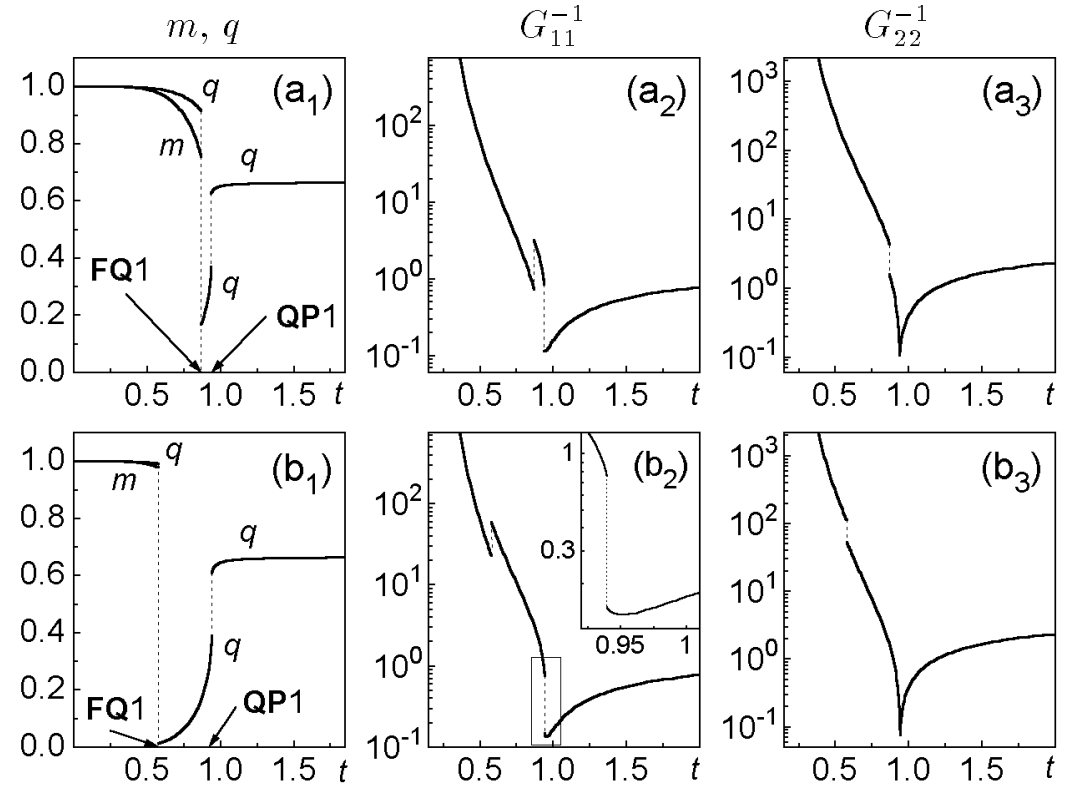

Figure 16. The temperature dependences of $m, q$ and CFs $G_{11}^{-1}(\vec{q}=0)$, $G_{22}^{-1}(\vec{q}=0)$ at $k^{\prime}=2.95$ for different values of parameter $d:(\mathrm{a})-d=-11.84 ; \quad(\mathrm{b})-d=-11.849$.
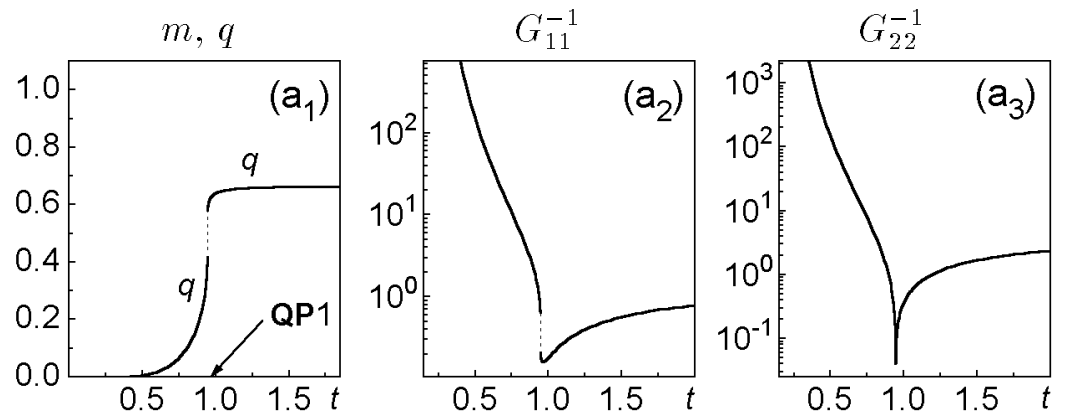

Figure 17. The temperature dependences of $m, q$ and CFs $G_{11}^{-1}(\vec{q}=0)$, $G_{22}^{-1}(\vec{q}=0)$ at $k^{\prime}=2.95$ for $d=-11.86$.

(see figure $7(\mathrm{c})$ ) appear in the paramagnetic phase. At smaller values of $d G_{22}^{-1}\left(t_{c}\right)$ decreases with a discontinuity (see figure $7(\mathrm{~d})$ ). For illustration, we depict in figure 8 the temperature dependences of $G_{11}^{-1}, G_{22}^{-1}$ at $k^{\prime}=1.0$ (the changes in the temperature curves of single-site CFs and pair CFs with $d$ are similar at $k^{\prime}=0$ and $\left.k^{\prime}=1.0\right)$ which correspond to two latter cases for $k^{\prime}=0$ (figures $7(\mathrm{c}), 7(\mathrm{~d})$ ). At $k^{\prime}=2.0 G_{22}^{-1}\left(t_{c}\right)$ decreases with a discontinuity (see figure 10(b)) when $d$ is decreased. Then, minima of $G_{11}^{-1}(t)$ (see figure 10(c)), and then, of $G_{22}^{-1}(t)$ (see figure 10(d)) appear in the $\mathbf{P}$-phase. At smaller values of $d$ at first $G_{11}^{-1}\left(t_{c}\right)$ has upward step (see figure 10(e)), but then again this step become downward (see figure 10(f)). That is, in the vicinity of the regions IV at $k^{\prime}=0.0,1.0,2.0$ the behaviour 

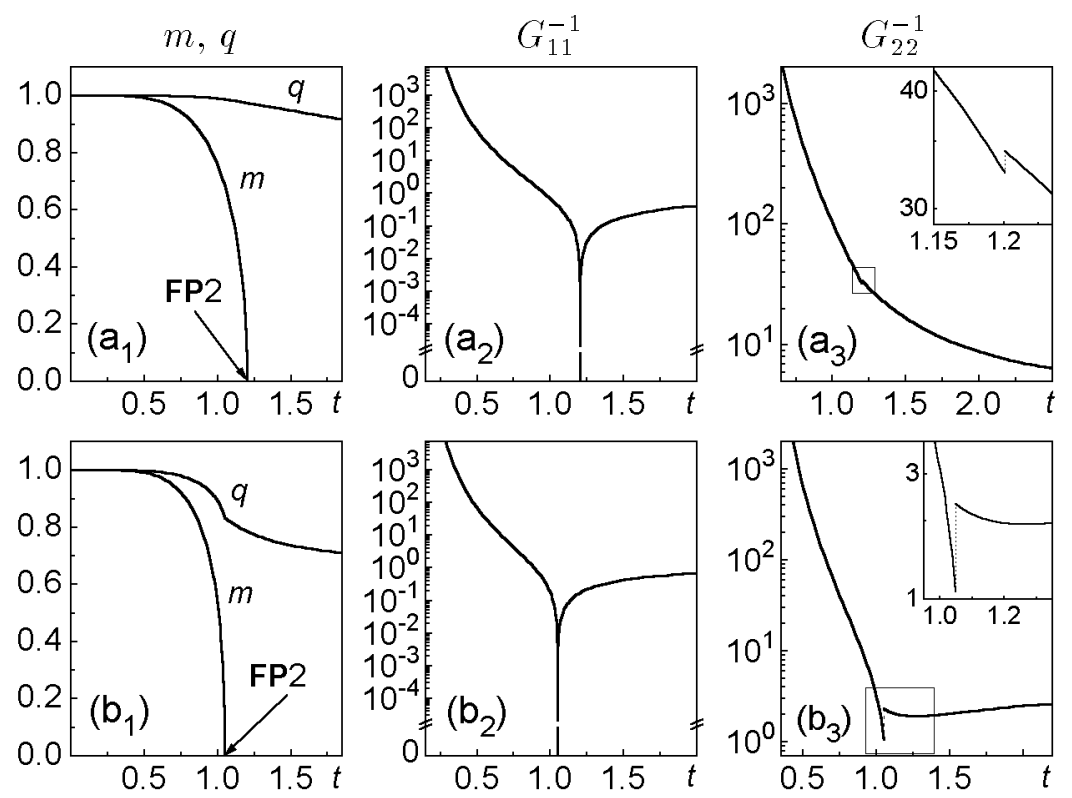

Figure 18. The temperature dependences of $m, q$ and CFs $G_{11}^{-1}(\vec{q}=0)$, $G_{22}^{-1}(\vec{q}=0)$ at $k^{\prime}=3.2$ for different values of parameter $d$ : (a) $-d=-5.0 ; \quad$ (b) $-d=-12.0$.
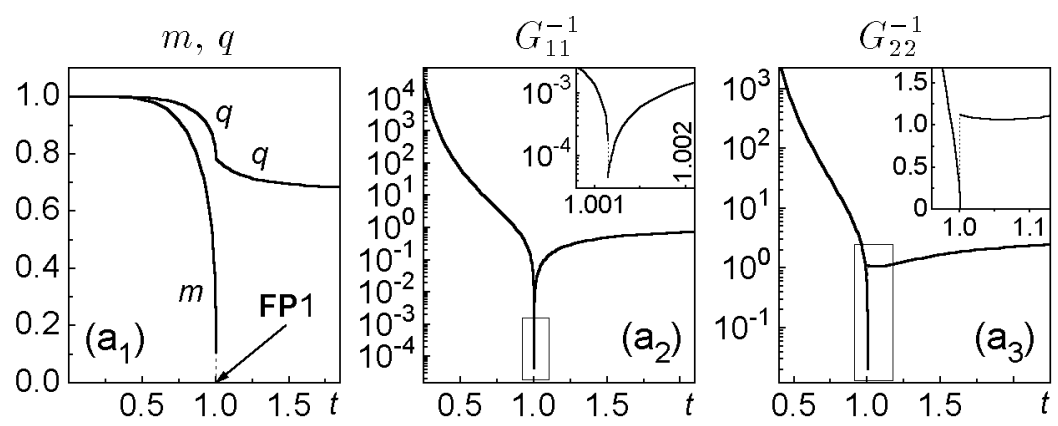

Figure 19. The temperature dependences of $m, q$ and CFs $G_{11}^{-1}(\vec{q}=0)$, $G_{22}^{-1}(\vec{q}=0)$ at $k^{\prime}=3.2$ for $d=-12.47$.
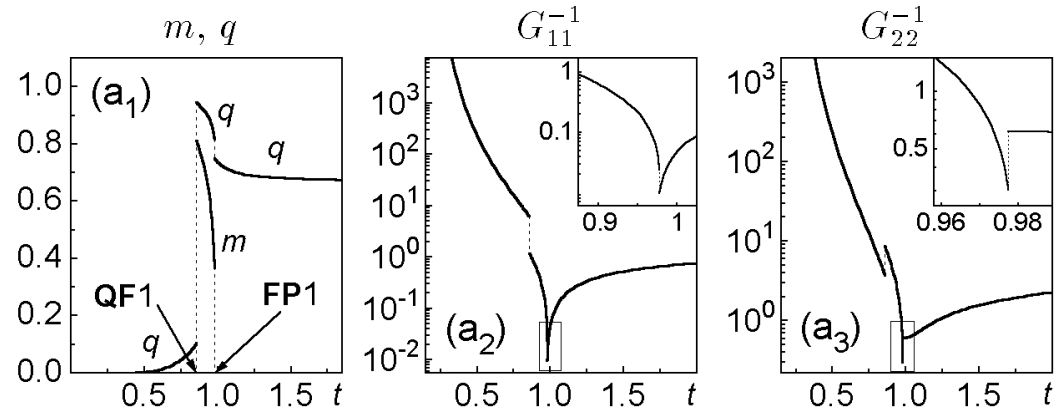

Figure 20. The temperature dependences of $m, q$ and CFs $G_{11}^{-1}(\vec{q}=0)$, $G_{22}^{-1}(\vec{q}=0)$ at $k^{\prime}=3.2$ for $d=-12.63$. 

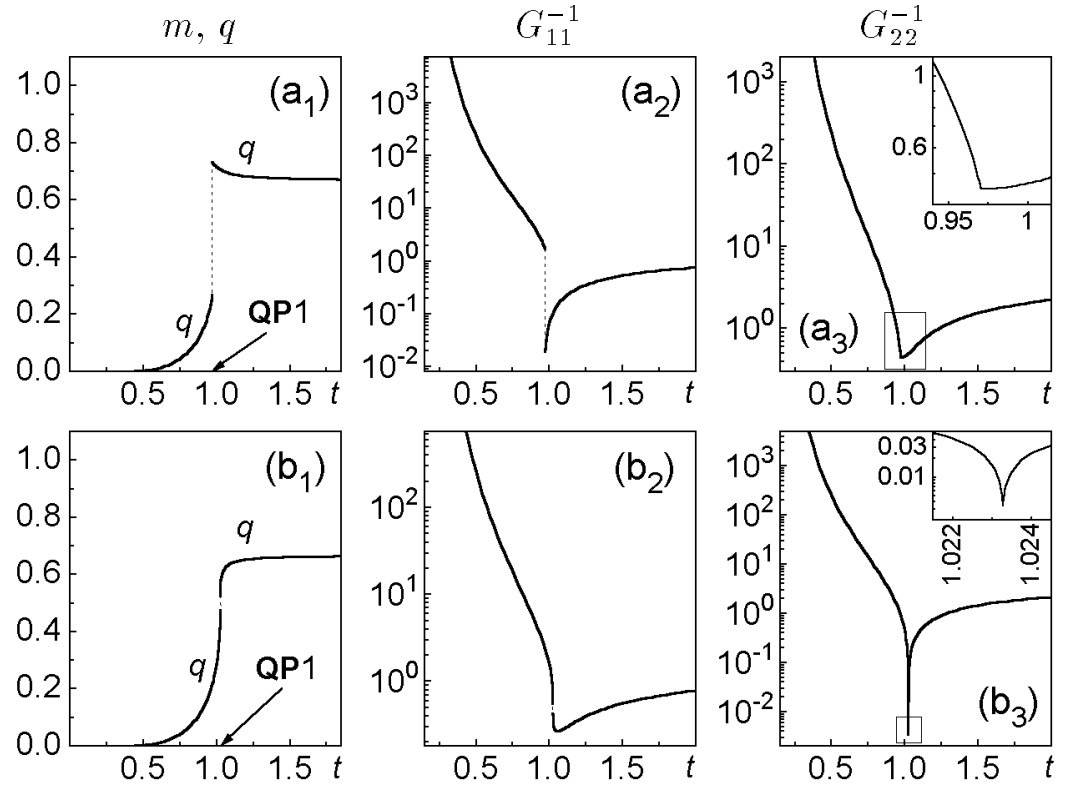

Figure 21. The temperature dependences of $m, q$ and CFs $G_{11}^{-1}(\vec{q}=0)$, $G_{22}^{-1}(\vec{q}=0)$ at $k^{\prime}=3.2$ for different values of parameter $d$ : (a) $-d=-12.673 ; \quad$ (b) $-d=-12.8$.
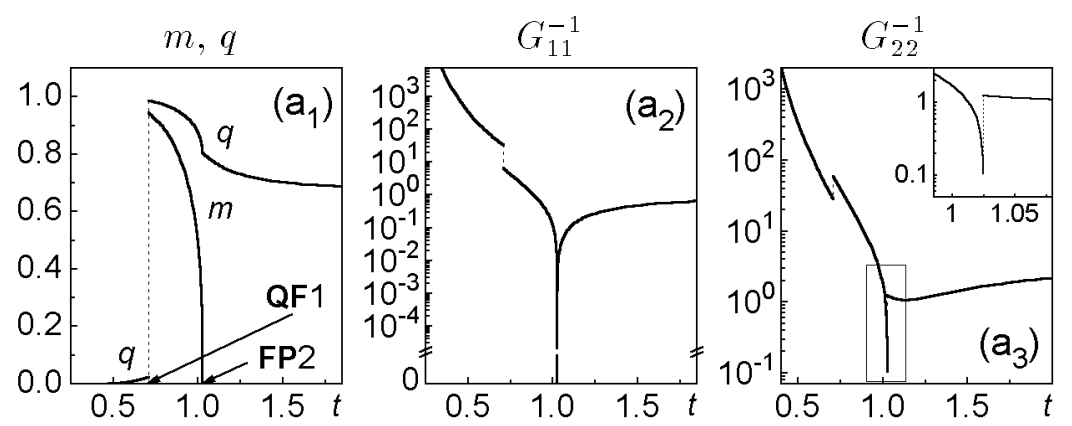

Figure 22. The temperature dependences of $m, q$ and CFs $G_{11}^{-1}(\vec{q}=0)$, $G_{22}^{-1}(\vec{q}=0)$ at $k^{\prime}=3.44$ for $d=-13.34$.

of CFs $G_{11}^{-1}(t)$ and $G_{22}^{-1}(t)$ is qualitatively similar (see figures $7(\mathrm{~d}), 8(\mathrm{~b})$, $10(\mathrm{f}))$. The changes in behaviour of CFs with decreasing of $d$ at $k^{\prime}=2.6$ and $k^{\prime}=2.88$ are at first the same as those at $k^{\prime}=2.0$ (see figures $10(\mathrm{~b})$, 11(b), 12(b), 10(c), 11(c), 12(c)). Near the region IV $G_{11}^{-1}\left(t_{c}\right)$ increases with a discontinuity at $k^{\prime}=2.6$ (see fig 11(d)). Behaviour of $G_{11}^{-1}(t)$ near the region VII at $k^{\prime}=2.88$ is similar to that at $k^{\prime}=2.6$ near the region $\mathrm{IV}$, but CF $G_{22}^{-1}(t)$ has no minimum in the paramagnetic phase (see figures $11(\mathrm{~d}), 12(\mathrm{~d}))$. Between the regions VII and IV a minimum of $G_{22}^{-1}\left(t>t_{c}\right)$ appears; the behaviour of $G_{11}^{-1}(t)$ and $G_{22}^{-1}(t)$ is the same as at $k^{\prime}=2.6$ near the region IV (see figures $11(\mathrm{~d}), 14)$ ). At $k^{\prime}=2.95$ the region III is rather narrow, therefore, only $G_{22}^{-1}(t)$ behaviour is changes with a decrease in $d$ 

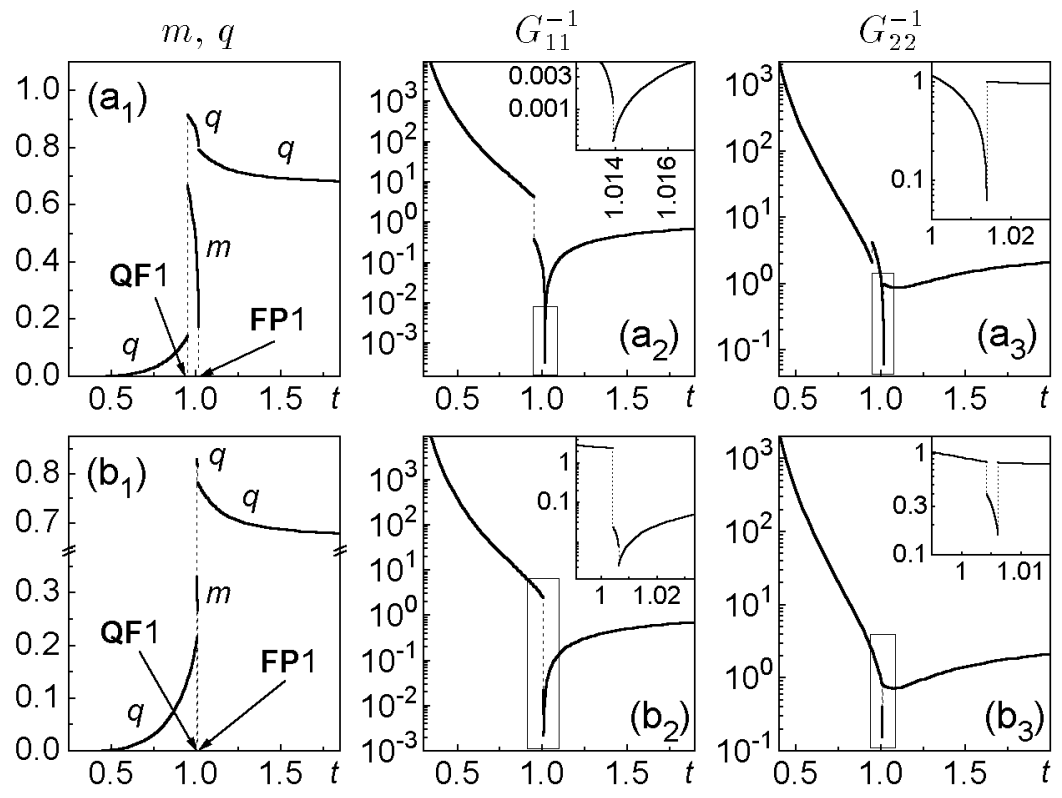

Figure 23. The temperature dependences of $m, q$ and CFs $G_{11}^{-1}(\vec{q}=0)$, $G_{22}^{-1}(\vec{q}=0)$ at $k^{\prime}=3.44$ for different values of parameter $d:($ a) $-d=-13.42 ; \quad$ (b) $-d=-13.475$.
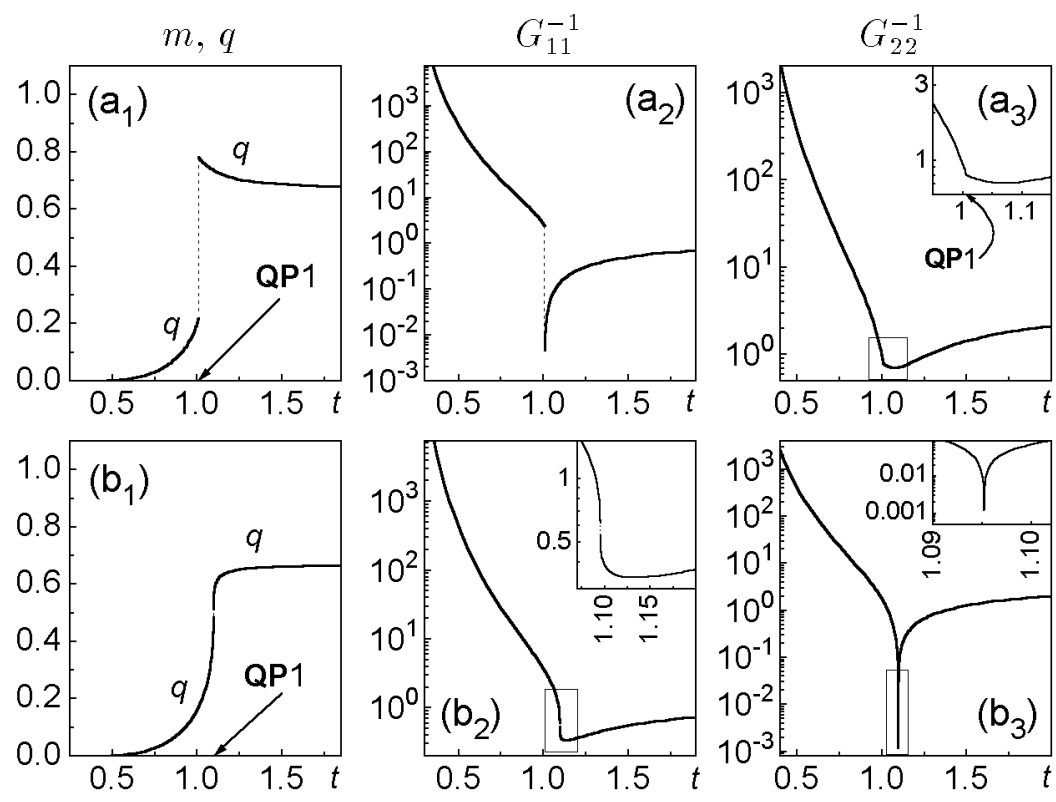

Figure 24. The temperature dependences of $m, q$ and CFs $G_{11}^{-1}(\vec{q}=0)$, $G_{22}^{-1}(\vec{q}=0)$ at $k^{\prime}=3.44$ for different values of parameter $d:(\mathrm{a})-d=-13.48 ; \quad$ (b) $-d=-13.696$. 

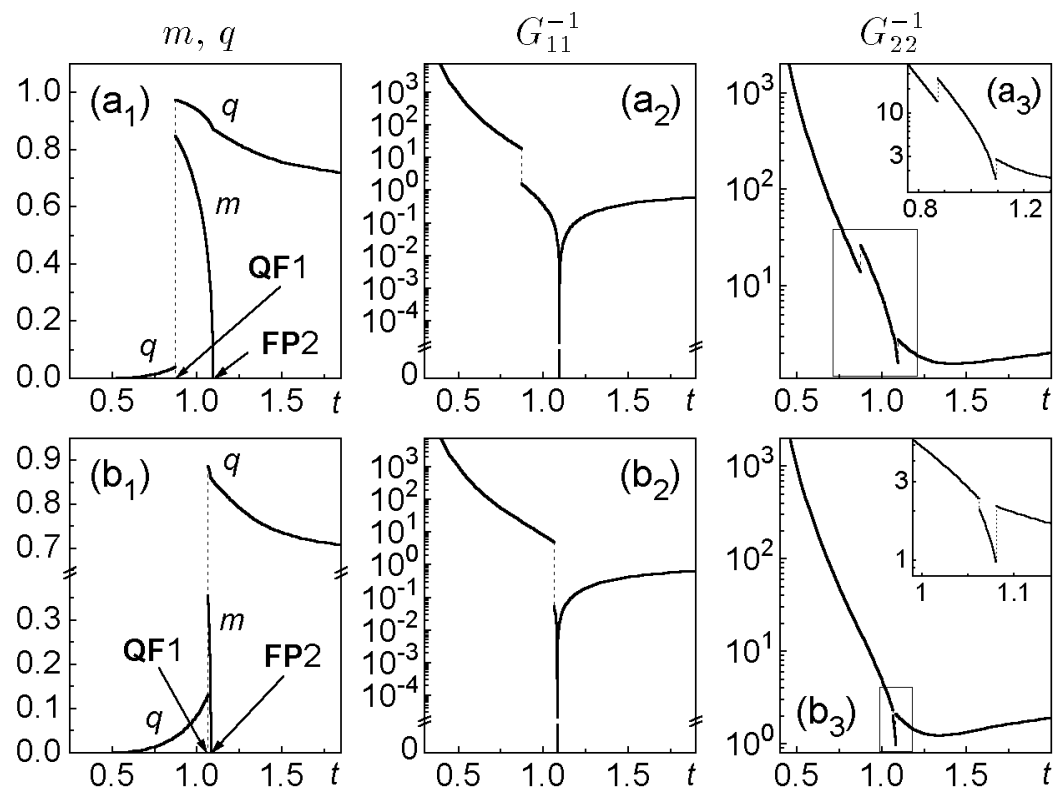

Figure 25. The temperature dependences of $m, q$ and CFs $G_{11}^{-1}(\vec{q}=0)$, $G_{22}^{-1}(\vec{q}=0)$ at $k^{\prime}=4.0$ for different values of parameter $d$ : (a) $-d=-15.1 ;$ (b) $-d=-15.3$.

in this region (see figure $15(\mathrm{~b})$ ). Let us note that the changes in $G_{22}^{-1}(t)$ behaviour with $d$ are the same as those at $k^{\prime}=2.0,2.6,2.88$ in the region III near the region II (see figures 10(b), 11(b), 12(b)). The behaviour of $G_{11}^{-1}(t)$ and $G_{22}^{-1}(t)$ at $k^{\prime}=3.2$ in the region VI (between the regions $\mathrm{V}$ and $\mathrm{I}$ ) is qualitatively different from that at $k^{\prime}=0.0,1.0,2.0,2.6,2.88,2.95$ near the region II (see figures $7(\mathrm{a}), 10(\mathrm{a}), 11(\mathrm{a}), 12(\mathrm{a}), 15(\mathrm{a})$ ). Here $G_{22}^{-1}\left(t>t_{c}\right)$ (see figure 19) has a mimimum (such behaviour of $G_{22}^{-1}(t)$ in the paramagnetic phase remains starting from the region II (see figure 18(b)). When the single-ion anisotropy is decreased (moving from the region II in regions III and VI) a minimum of $G_{22}^{-1}\left(t>t_{c}\right)$ dissappers (see figure 20), and the behaviour of $G_{11}^{-1}(t)$ and $G_{22}^{-1}(t)$ becomes the same as at $k^{\prime}=0.0,1.0,2.0$, $2.6,2.88,2.95$ near the region II (see figures $7(\mathrm{a}), 10(\mathrm{a}), 11(\mathrm{a}), 12(\mathrm{a}), 15(\mathrm{a})$ ).

Let us consider now the behaviour of the inverse correlation functions $G_{11}^{-1}(t), G_{12}^{-1}(t), G_{22}^{-1}(t)$ at the phase transition $\mathbf{Q P} 1$ in regions I and VII (see figures $13,16,17,21,24,26)$. Here, the inverse $\mathrm{CF} G_{12}^{-1}(t)$ is infinitely large both in phases $\mathbf{Q}$ and $\mathbf{P}$. The inverse $\mathrm{CF} G_{11}^{-1}(t)$ has discontinuity, and $G_{11}^{-1}\left(t_{c}-1\right)>G_{11}^{-1}\left(t_{c}+0\right) ; G_{11}^{-1}\left(t<t_{c}\right)$ decreases and $G_{11}^{-1}\left(t>t_{c}\right)$ can either increase or first decrease and then increase. The inverse $\mathrm{CF} G_{22}^{-1}(t)$ has a cusp in the transition point. At $k^{\prime}=2.88$ in the entire region VII the inverse $\mathrm{CF} G_{11}^{-1}(t)$ has a minimum in the paramagnetic phase (see figure 13). At $k^{\prime}=2.95,3.2,3.44,4.0$, the $G_{11}^{-1}\left(t>t_{c}\right)$ increases (see figures 16(a), 21(a), 24(a), 26(a), 26(b)); a minimum of $G_{11}^{-1}\left(t>t_{c}\right)$ appears if $d$ is decreased (see figures 16(b), 21(b), 24(b), 26(c)). Let us also note that almost always if $q(t)$ increases in the paramagnetic phase then $G_{11}^{-1}\left(t>t_{c}\right)$ has a minimum 

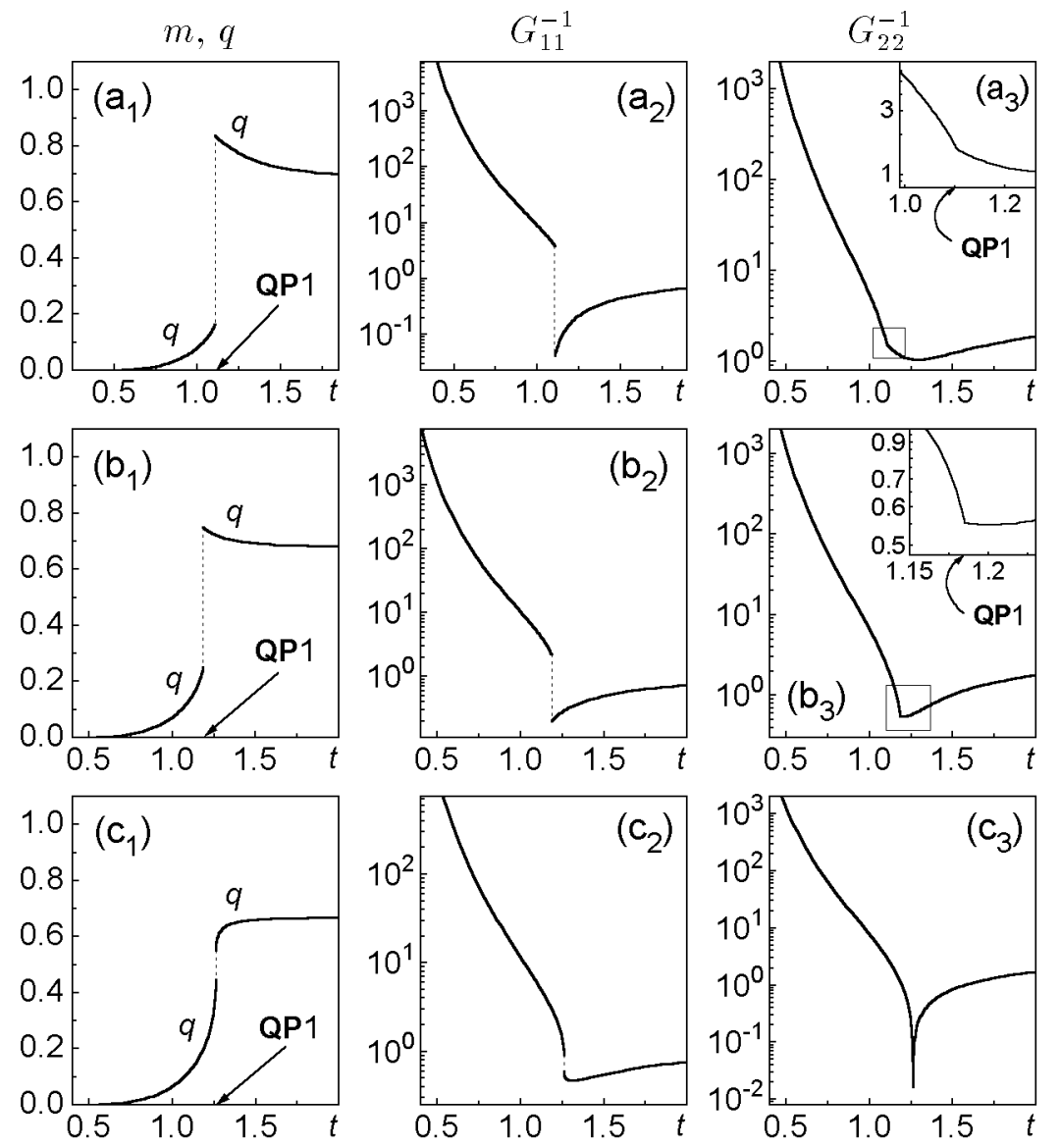

Figure 26. The temperature dependences of $m, q$ and CFs $G_{11}^{-1}(\vec{q}=0)$, $G_{22}^{-1}(\vec{q}=0)$ at $k^{\prime}=4.0$ for different values of parameter $d$ : (a) $-d=-15.4 ; \quad$ (b) $-d=-15.6 ; \quad$ (c) $-d=-15.79$.

(see figures 13, 16(b), 17, 21(b), 24(b), 26(c)), and if $q\left(t>t_{c}\right.$ ) decreases then $G_{11}^{-1}\left(t>t_{c}\right)$ increases (see figures 21(a), 24(a), 26(a), 26(b)). However, it is possible that even if $q(t)$ increases in $\mathbf{P}, G_{11}^{-1}\left(t>t_{c}\right)$ increases has

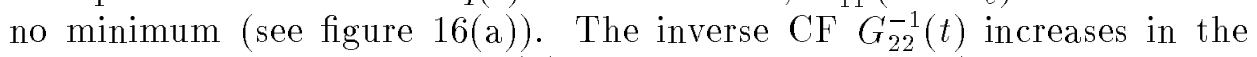
paramagnetic phase at small $k^{\prime}$ (see figures $13,16,17,21$ ). At large values of $k^{\prime}$ in the close proximity to the region $\mathrm{V}$, the $G_{22}^{-1}(t)$ decreases before the cusps at the transition point and after it and starts to increase only at higher temperatures (see figures 24, 26).

At the transition $\mathbf{F Q 1}$ (region VII), the inverse $\mathrm{CF}_{12}^{-1}(t)$ has an infinite discontinuity $\left(G_{12}^{-1}\left(t_{c}-0\right)>0, G_{12}^{-1}\left(t_{c}+0\right)=\infty ; G_{12}^{-1}\left(t<t_{c}\right)\right.$ decreases $)$, whereas a discontinuity the inverse CFs $G_{11}^{-1}(t)$ and $G_{22}^{-1}(t)$ if finite. In phases $\mathbf{F}, \mathbf{Q}$ inverse CFs $G_{11}^{-1}(t)$ and $G_{22}^{-1}(t)$ decrease, and $G_{11}^{-1}\left(t_{c}-0\right)<$ $G_{11}^{-1}\left(t_{c}+0\right), G_{22}^{-1}\left(t_{c}-0\right)>G_{22}^{-1}\left(t_{c}+0\right)$ (see fig 13,16$)$.

At the transition $\mathbf{Q F} 1$ (regions $\mathrm{V}, \mathrm{VI}$ ) the inverse $\mathrm{CF} G_{12}^{-1}(t)$ has an infinite discontinuity $\left(G_{12}^{-1}\left(t_{c}-0\right)=\infty, G_{12}^{-1}\left(t_{c}+0\right)>0 ; G_{12}^{-1}(t)\right.$ decreases 
in the phase $\mathbf{F}$ ), whereas a discontinuity of $G_{11}^{-1}(t)$ and $G_{22}^{-1}(t)$ is finite, and $G_{11}^{-1}\left(t_{c}-0\right)>G_{11}^{-1}\left(t_{c}+0\right)$. In phases $\mathbf{Q}$ and $\mathbf{F}$, inverse $C F s G_{11}^{-1}(t)$ and $G_{22}^{-1}(t)$ decreases (see figures $20,22,23,25$ ). At enough small values of $k^{\prime}$ and arbitrary $d$ (see figure 20), and also at longer values of $k^{\prime}$ and enough large $d$ (see figures 22, 23(a), 25(a)) $G_{22}^{-1}\left(t_{c}-0\right)<G_{22}^{-1}\left(t_{c}+0\right)$. At large $k^{\prime}$ and small $d$ (see figures $23(\mathrm{~b}), 25(\mathrm{~b})) G_{22}^{-1}\left(t_{c}-0\right)>G_{22}^{-1}\left(t_{c}+0\right)$.

\section{References}

[1] Blume M., Emery V.J., Griffiths R.B. Ising model for the $\lambda$-transition and phase separation in $\mathrm{He}^{3}-\mathrm{He}^{4}$ mixtures. // Phys. Rev. A., 1971, vol. 4, No 3, p. 1071-1077.

[2] Sivardiere J. Critical and multicritical points in fluids and magnets // Lecture' Notes in physics. Static critical phenomena in inhomogeneous systems. Proceedings, Karpacz. 1984, p. 247-289.

[3] Sorokov S.I., Levitskii R.R., Baran O.R. Two-site cluster approximation for Ising's models with arbitrary value of spin. I. The Blume-Capel-Griffiths model. // Lviv, 1993, 47p. (Preprint / Acad. Sci. Ukr. Inst.Cond.Mat.Phys.; ICMP-93-1U). (In Ukrainian).

[4] Wang Y., Lee F. Linked-cluster series analysis of the Blume-Capel model. // The American Physical Society, 1984, vol. 29, No 9, p. 5156-5164.

[5] Smart J.S. Effective field theories of magnetism. -Philadelphia-London: W.B.Saunders company, 1996.

[6] Vaks V.G. Introduction to the microscopic theory of ferroelectrics. -M.: Nauka, 1973, 327p. (In Russian).

[7] Vaks V.G., Zinenko V.I., Shneider V.E. Microscopic theories of the structural phase transitions of the order-disorder type in crystals. // Usp.Fiz.Nauk, 1983, vol. 141, No 4, p. 626-673. (In Russian).

[8] Kubo P. Generalized cumulant expansion method. // J. Phys. Soc. Japan, 1962, vol. 17, No 7, p. 1100-1120.

[9] Strieb B., Callen H.B. Cluster expansion for the Heisenberg ferromagnet. // Phys. Rev., 1963, vol. 130, No 5, p. 1798-1808.

[10] Iwashita T., Uryû N. Ising Ferromagnet with biquadratic exchange interaction and uniaxial anisotropy. // Phys. Stat. Sol. (b), 1986, vol. 137, p. 65-72.

[11] Kaneyoshi T., Sarmento E.F. The application of the differential operator method to the Blume-Emery-Griffiths model. // Phys. A, 1988, vol. 152, p. $343-358$.

[12] Tucker J.W. Two-site cluster theory for the spin-one Ising model. // J. Magn. Magn. Mat., 1990, vol. 87, p. 16-22.

[13] Chakraborty K.G. Effective-field model for a spin-1 Ising system with dipolar and quadrupolar interactions. // Phys. Rev. B, 1984, vol. 29, No 3, p. 14541457.

[14] Siqueira A.F., Fittipaldi I.P. On the phase transition in the Blume-Capel model. // Phys. Stat. Sol. (b), 1983, vol. 119, p. K31-K36.

[15] Tamura I., Kaneyoshi T. Ising ferromagnets with random anisotropy. // Prog. Theor. Phys., 1981, vol. 66, No 5, p. 1892-1894.

[16] Taggart B.G. Correlation effective field approximation for the Ising model. // Physica A, 1982, vol. 113A, p. 535-545.

[17] Takahashi K., Tanaka M. Spin-one Ising model with bilinear and biquadratic interactions. II. Phase diagram. // J. Phys. Soc. Japan, 1980, vol. 48, No 5, p. 1423-1428.

[18] Kasono K., Ono I. Re-entrant phase transitions of the Blume-Emery-Griffiths model. // Z. Phys. B - Condensed Matter, 1992, vol. 88, p. 205-212.

[19] Saul D., Wortis M., Stauffer D. Tricritical behavior of the Blume-Capel model. // Phys. Rev. B, 1974, vol. 9, No 11, p. 4964-4980.

[20] De Alcantara Bonfim O.F., Obcemea C.H. Reentrant behaviour in Ising models with biquadratic exchange interaction. // Z. Phys. B - Condensed Matter, 
1986, vol. 64, p. 469-471

[21] Booth R.J.C., Lu Hua, Tucker J.W., Care C.M., Halliday I. Monte Carlo study of the BEG model on a honeycomb lattice. // J. Magn. Magn. Mat., 1993, vol. 128 , p. 117-123.

[22] Sorokov S.I., Levitskii R.R., Baran O.R. The study of Ising model within cluster expansion method.// Lviv, 1992, 47p. (Preprint / Acad. Sci. Ukr. Inst.Cond.Mat.Phys.; ICMP-92-18U). (In Ukrainian).

[23] Yukhnovskii I.R., Levitskii R.R., Sorokov S.I. Thermodynamics and distribution functions of Ising model. Two-site cluster approximation. // Kiev, 1986, 33p. (Preprint / Acad. Sci. Ukr.SSR Inst.Theor.Phys.; ITP-86-142R). (In Russian).

[24] Sorokov S.I., Levitskii R.R., Baran O.R. The Blume-Capel-Griffiths model within two-particle cluster approximation. Numerical calculations. // Lviv, 1995, 45p. (Preprint / Acad. Sci. Ukr. Inst.Cond.Mat.Phys.; ICMP-95-9U). (In Ukrainian).

[25] Chen H., Levy P. Dipole and quadrupole phase transitions in spin-1 models. // Phys. Rev. B, 1973, vol. 7, No 9, p. 4267-4283.

\title{
НАБЛИЖЕННЯ ДВОЧАСТИНКОВОГО КЛАСТЕРА ДЛЯ ІЗІНГІВСЬКИХ МОДЕЛЕЙ З ДОВІЛЬНИМ ЗНАЧЕННЯМ СПІНА. КОРЕЛЯЩІЙНІ ФУНКЦІЇ МОДЕЛІ БЛЮМА-ЕМЕРІ-ГРІФІТСА
}

\author{
С.І.Сороков, Р.Р.Левицький, О.Р.Баран
}

В наближенні двочастинкового кластера досліджуються Ізінгівські моделі з довільним значенням спіна. Для цих моделей у випадку гіперкубічних граток отримано вирази для парних кореляційних функцій у $\vec{q}$-просторі. У випадку простої кубічноі гратки для моделі Блюма-Емері-Гріфітса $(S=1) 3$ білінійною $K$, біквадратичною $K^{\prime}$ взаємодіями та одноіонною анізотропією $D$ побудована проекція фазової діаграми на прощину $D / K-K^{\prime} / K$. При різному наборі значень параметрів $D / K$ та $K^{\prime} / K$ отримані температурні залажності $\left\langle S^{z}\right\rangle,\left\langle\left(S^{z}\right)^{2}\right\rangle$ та парних кореляційних функцій при $\vec{q}=0$. 
